Corrected: Author correction

ARTICLE

DOI: $10.1038 / s 41467-018-05459-z$

\title{
Pathogen-derived HLA-E bound epitopes reveal broad primary anchor pocket tolerability and conformationally malleable peptide binding
}

Lucy C. Walters ${ }^{1}$, Karl Harlos², Simon Brackenridge (10 1, Daniel Rozbesky², Jordan R. Barrett1, Vitul Jain², Thomas S. Walter (1) ${ }^{2}$, Chris A. O'Callaghan ${ }^{3}$, Persephone Borrow ${ }^{1}$, Mireille Toebes ${ }^{4}$, Scott G. Hansen ${ }^{5}$, Jonah B Sacha ${ }^{5}$, Shaheed Abdulhaqq ${ }^{5}$, Justin M. Greene ${ }^{5}$, Klaus Früh ${ }^{5}$, Emily Marshall ${ }^{5}$, Louis J. Picker ${ }^{5}$, E. Yvonne Jones (iD ${ }^{2}$, Andrew J. McMichael ${ }^{1} \&$ Geraldine M. Gillespie $^{1}$

Through major histocompatibility complex class la leader sequence-derived (VL9) peptide binding and CD94/NKG2 receptor engagement, human leucocyte antigen E (HLA-E) reports cellular health to NK cells. Previous studies demonstrated a strong bias for VL9 binding by HLA-E, a preference subsequently supported by structural analyses. However, Mycobacteria tuberculosis (Mtb) infection and Rhesus cytomegalovirus-vectored SIV vaccinations revealed contexts where HLA-E and the rhesus homologue, Mamu-E, presented diverse pathogenderived peptides to $\mathrm{CD}^{+}{ }^{+} \mathrm{T}$ cells, respectively. Here we present crystal structures of HLA-E in complex with HIV and Mtb-derived peptides. We show that despite the presence of preferred primary anchor residues, HLA-E-bound peptides can adopt alternative conformations within the peptide binding groove. Furthermore, combined structural and mutagenesis analyses illustrate a greater tolerance for hydrophobic and polar residues in the primary pockets than previously appreciated. Finally, biochemical studies reveal HLA-E peptide binding and exchange characteristics with potential relevance to its alternative antigen presenting function in vivo.

\footnotetext{
${ }^{1}$ Nuffield Department of Medicine Research Building, Roosevelt Drive, Nuffield Department of Medicine, University of Oxford, Oxford OX3 7FZ, UK. ${ }^{2}$ Division of Structural Biology, Wellcome Centre for Human Genetics, Roosevelt Drive, University of Oxford, Oxford OX3 7BN, UK. ${ }^{3}$ Henry Wellcome Building for Molecular Physiology, University of Oxford, Oxford OX3 7BN, UK. ${ }^{4}$ Department Molecular Oncology and Immunology, B6 Plesmanlaan 121, Amsterdam 1066CX, The Netherlands. ${ }^{5}$ Vaccine and Gene Therapy Institute and Oregon National Primate Research Center, Oregon Health \& Science University, Beaverton, OR 97006, USA. These authors contributed equally: Andrew J. McMichael, Geraldine M. Gillespie. Correspondence and requests for materials should be addressed to A.J.M. (email: andrew.mcmichael@ndm.ox.ac.uk) or to G.M.G. (email: geraldine.gillespie@ndm.ox.ac.uk)
} 
uman leucocyte antigen $\mathrm{E}$ (HLA-E) is a non-classical MHC class Ib molecule homologous to H-2 Qa-1 in mice and Mamu-E in rhesus macaques $(\mathrm{RM})^{1-4}$. The two common human allotypes, HLA-E ${ }^{\star} 01: 01$ and HLA-E ${ }^{\star} 01: 03$, are essentially monomorphic, differing by a single amino acid substitution (Arg or Gly) at position 107, situated on a loop outside the peptide binding groove (PBG). Although relative expression is higher for HLA-E ${ }^{\star} 01: 03$, both subtypes are present on the cell surface at lower levels than classical HLA-A or HLA-B molecules ${ }^{3,4}$. HLA-E exhibits preferential binding to a highly conserved set of nonameric signal (VL9) peptides derived from the leader sequence of HLA-A, B, C or G molecules ${ }^{5-7}$. Primary anchor residues are largely conserved among VL9 peptides: the canonical position 2 Met and position 9 Leu are accommodated by the primary $\mathrm{B}$ and $\mathrm{F}$ pockets, respectively. However, a position 2 Thr, present in a subset of HLA-B molecules, results in lower binding affinity to HLA-E and consequently, reduced surface expression $^{7-9}$. VL9 peptide-bound HLA-E complexes engage the natural killer (NK) cell inhibitory receptor, CD94-NKG2A, thereby protecting healthy cells from NK cell-mediated lysis ${ }^{6,9}$. HLA-E also binds the NK cell activating receptor, CD94-NKG2C, although such interactions are of lower affinity ${ }^{10}$. Whilst VL9 peptide binding and NK cell regulation are ostensibly its primary function, HLA-E, and its rhesus and murine homologues, can present peptides from microbial and autologous sources to CD8 ${ }^{+}$ $\mathrm{T}$ cells $\mathrm{s}^{3,11-21}$. In Mycobacterium tuberculosis (Mtb) infection, multiple mycobacterial peptides have been shown to stimulate HLA-E restricted $\mathrm{CD}^{+}{ }^{+} \mathrm{T}$ cells ${ }^{15}$. Similarly, Mamu-E restricted $\mathrm{CD}^{+} \mathrm{T}$ cell responses are elicited in $\mathrm{RM}$ by an experimental rhesus cytomegalovirus (RhCMV68-1) vaccine, recombinant for simian immunodeficiency virus (SIV) genes ${ }^{13}$. In both cases, the antigen presentation pathways are atypical, arising from Mtbinfected phagolysosomes in macrophage ${ }^{22}$ or vector-mediated disruption in RhCMV68-1-vaccinated macaques ${ }^{13}$. Remarkably, RhCMV68-1 stimulated SIV epitopes are particularly diverse with no simple sequence motif ${ }^{13}$, implying unexpectedly permissive peptide binding by Mamu- $\mathrm{E}^{23}$. These broad, Mamu-E restricted $\mathrm{CD}^{+} \mathrm{T}$ cell responses have been implicated as immune correlates of protection in RhCMV68-1 SIV vaccine studies ${ }^{13}$.

Here we explore pathogen-derived peptide binding to HLA-E from a structural and biochemical perspective. Through combined sandwich enzyme-linked immunosorbent assay (ELISA)based and single-chain trimer approaches we confirm that the HLA-E peptide repertoire is broad. Via the mutagenesis and structural analysis of pathogen-derived peptides we also demonstrate an increased binding capacity of the primary pockets, with a greater breadth of tolerated anchor residues than originally reported for HLA-E-binding peptides ${ }^{5,23-26}$. This diversity also extends to the conformation of HLA-E-bound peptide which we show can differ dramatically from canonically orientated VL9, even in the presence of preferred primary anchor residues. Finally, we demonstrate that HLA-E is relatively stable without added peptide, favouring both low-affinity peptide binding and peptide exchange. These characteristics likely favour promiscuous peptide sampling in vivo, especially when the peptide loading complex (PLC) is disrupted, or absent in a peripheral intracellular compartment.

\section{Results}

Pathogen-derived peptide binding to HLA-E $\mathrm{E}^{\star} 01: 03$. A microrefolding and sandwich ELISA-based approach enabled relative quantification of peptide binding affinity for HLA-E ${ }^{\star} 01: 03$. Two nonameric peptide panels were tested: the first included the HIVGag-derived, RMYSPTSIL (RL9HIV), a NetMHC predicted epitope $^{27}$ homologous to the SIVGag-derived RMYNPTNIL peptide (RL9SIV), which constituted one of the two supertopes recognised by $100 \%$ of protected macaques in RhCMV68-1 vaccine trials ${ }^{13}$. Furthermore, since RL9HIV ranked as a strong binder relative to previously reported HLA-E restricted microbial peptides in the micro-refolding ELISA ${ }^{25,28}$ (Fig. 1a) and elicited Mamu-E restricted CD8 ${ }^{+} \mathrm{T}$ cell responses in $\mathrm{RM}$ vaccinated with an HIVGag-insert RhCMV68-1 vector (Fig. 1b), it was selected for crystallographic analysis.

A selection of published Mtb-derived peptides ${ }^{15}$ constituted the second panel, of which four facilitated HLA-E*01:03 complex formation in the micro-refolding ELISA (Fig. 1c). One peptide, RLPAKAPLL (Mtb44), exhibited comparable binding to that of the MHC class Ia-derived VL9 positive control peptide. As Mtb44 also elicited Mamu-E-restricted $\mathrm{CD}^{+} \mathrm{T}$ cell responses in Bacillus Calmette-Guérin (BCG) vaccinated RM (Fig. 1d), it was pursued in crystallographic studies.

HLA-E ${ }^{\star}$ 01:03-Mtb44 structure. Previous crystal structures of HLA-E have been determined in complex with MHC class Ia leader sequence-derived VL9 peptides or HCMV UL40 proteinderived VL9 mimics ${ }^{8,10,23}$. Here we present the structure of HLA$\mathrm{E}^{\star} 01: 03$ bound to the Mtb-derived peptide, Mtb44 (RLPAKAPLL) (Fig. 2a). The complex packed in the P1 space group, diffracted to $2.1 \AA$ and was assigned the PDB ID, 6GH1 (Supplementary Table 1). Clear electron density was visible in the PBG into which the Mtb44 peptide was modelled (Fig. 2a). Despite sequence disparity, superposition of Mtb44 and canonical VL9 revealed strong conformational similarity in peptide positioning (Fig. 2b). Both peptides adopt the classical kinked orientation in which the backbone arches away from the groove floor projecting residues four and five (P4\&5) towards the solvent. Furthermore, eight of the nine canonical hydrogen $(\mathrm{H})$ bonds connecting peptide and heavy chain (HC) are conserved with $1 \mathrm{MHE}$. Formation of the Mtb44 P9-Leu [OXT] HLA-E Lys-146 [NZ] Hbond may counterbalance any loss of complex stability arising from the absent P5 H-bond, which connects VL9 to the a2 helix in 1MHE. Consistent with these structural observations and peptide binding assay data (Fig. 1 and Supplementary Table 2), Mtb44 complex stability is also underscored by thermal melt (Tm) analysis: Mtb44 shows a modest Tm increase $\left(\mathrm{Tm}=50.2^{\circ}\right.$ $\mathrm{C} \pm 0.3)$ compared to the HLA-A2 leader sequence-derived VL9 peptide $\mathrm{Tm}$ documented here $\left(\mathrm{Tm}=47.5^{\circ} \mathrm{C} \pm 0.4\right)$, and values previously reported for other MHC class Ia-derived leader sequence peptides 8,10

Analogous to previous VL9-bound structures, side chains of primary and secondary anchor residues in the Mtb44 complex project into their corresponding pockets towards the groove floor. Minimal repositioning of HLA-E-derived B pocket-lining residues suggests that $\mathrm{P} 2$ primary anchor Met to Leu substitution is well tolerated. Furthermore, the small side chains of P3-Pro and P6-Ala occupy the shallow D and C pockets, respectively.

HLA-E ${ }^{\star}$ 1:03-RL9HIV structure. Diffraction data from multiple isomorphous HLA-E ${ }^{\star} 01: 03-$ RL9HIV peptide crystals were merged in Xia2 yielding a 100\% complete dataset to $2.6 \AA$ resolution (Supplementary Table 1). The structure (PDB ID: 6GL1) was determined in the $\mathrm{C} 2$ space group with clear electron density visible in the PBG into which the RL9HIV peptide was modelled (Fig. 2d). RL9HIV positioning shows marked differences to the canonically orientated VL9 and Mtb44 peptides, in which P4 and 5 backbone arching ensures optimally positioned secondary anchor residues at P3, 6 and 7 for secondary $\mathrm{D}, \mathrm{C}$ and $\mathrm{E}$ pocket binding, respectively (Fig. 2e). RL9HIV exhibits an alternative, Cterminally shifted, kinked motif, wherein P6 and P7 arch away 
from the base of the groove, disrupting $\mathrm{C}$ and $\mathrm{E}$ pocket occupancy. The P6 RL9HIV and VL9 Ca atoms are separated by $4.2 \AA$ and the P7 C $\alpha$ atoms by $3.4 \AA$ (Fig. $2 \mathrm{~h}$ (i)), disrupting the ability of the RL9HIV P6-Thr and P7-Ser side chains to occupy their respective $\mathrm{C}$ and $\mathrm{E}$ pockets (Fig. 2f (i) and (ii)), and concomitantly rendering this region of the peptide more solvent exposed (Fig. 2h (ii)). However, the extended conformation between P1 and 5 of the RL9HIV peptide, arising from Cterminally shifted backbone arching, positions the P5-Pro Ca atom only $1.8 \AA$ from the VL9 P6-Thr Ca atom, with the two side chains deviating by as little as $1.2 \AA$, potentially permitting a degree of compensatory $\mathrm{C}$ pocket occupancy. Despite minimal Ca a

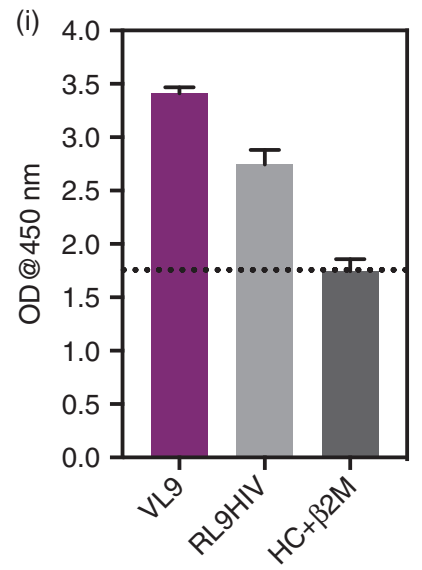

(ii)

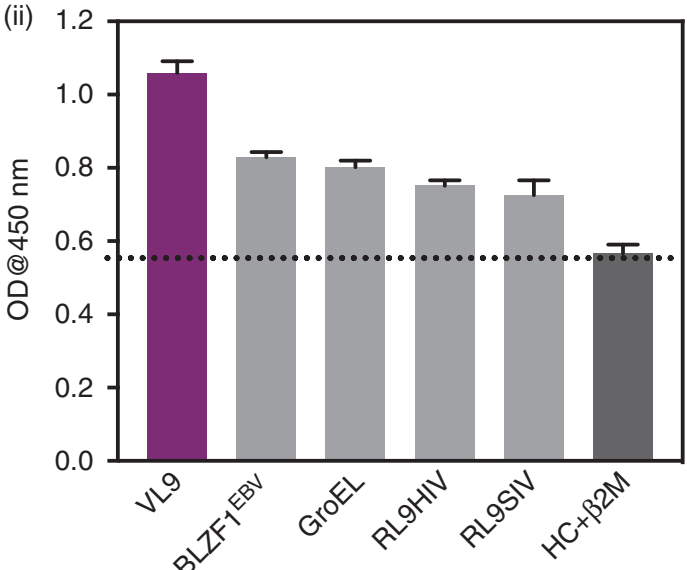

b

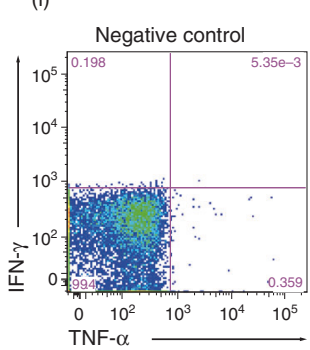

HIVgag-RL9 (RMYSPTSIL)
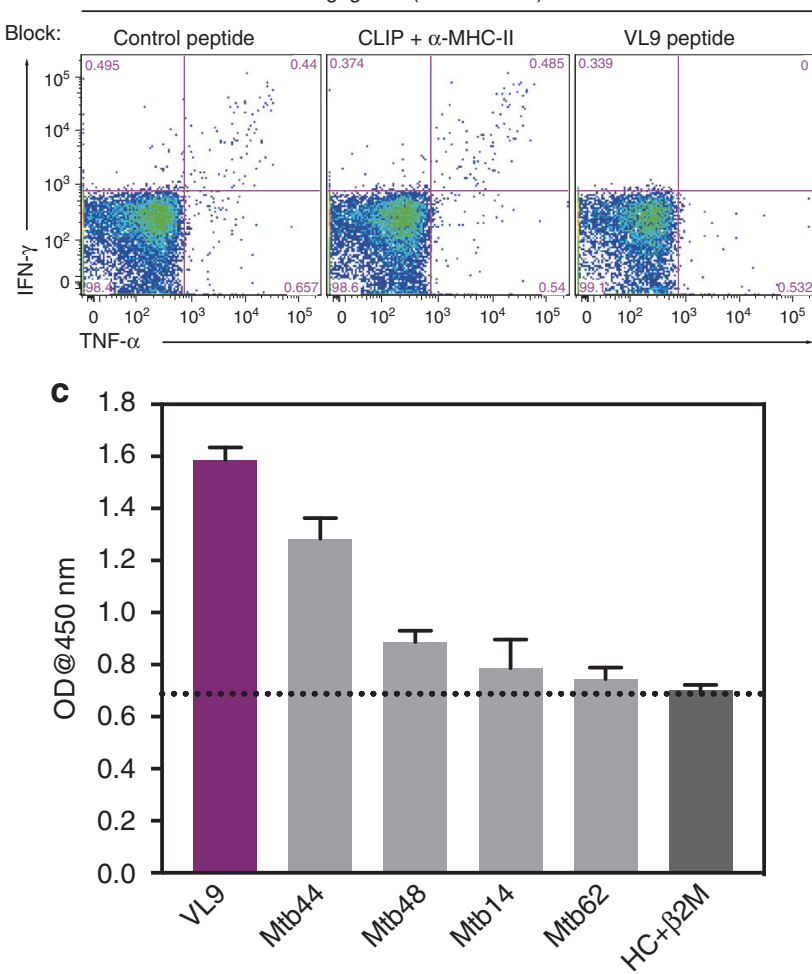

d

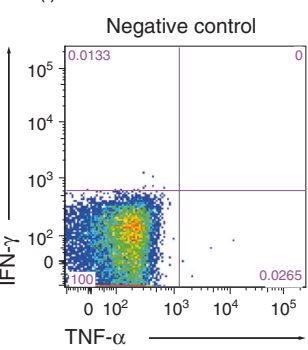

Mtb44 (RLPAKAPLL)

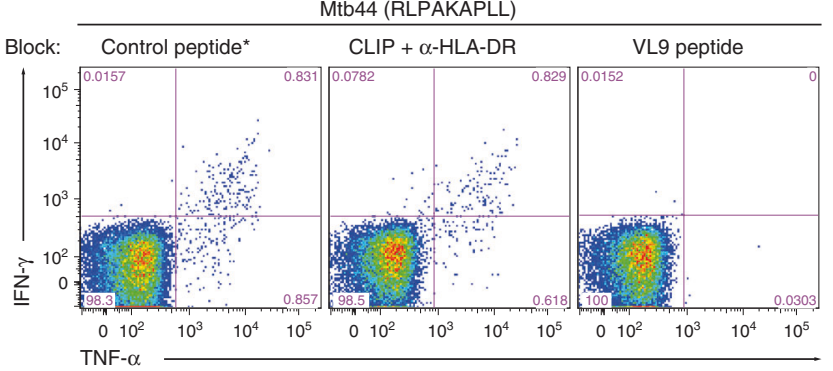

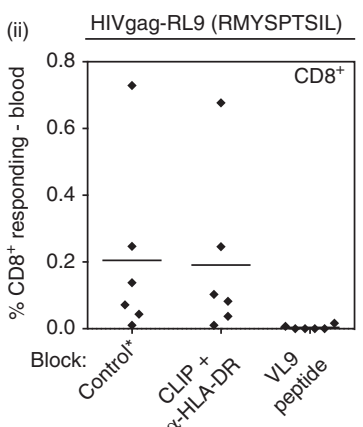

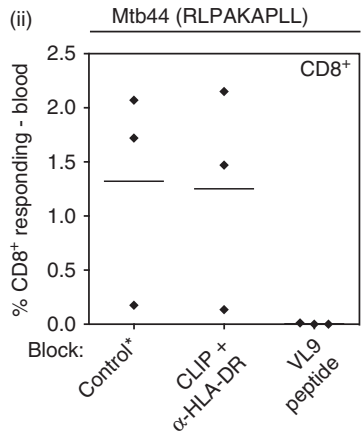


deviation facilitating optimal secondary anchor residue-pocket alignment, the P3-Tyr side chain is prohibitively large for shallow $\mathrm{D}$ pocket binding (Fig. $2 \mathrm{f}$ (iii)), instead projecting toward the $\alpha 2$ helix in the C-terminal direction of the peptide. This in turn triggers the D pocket lining residue, His-99, to adopt an alternative rotamer. An extensive, yet distinct, hydrogen-bonding network secures RL9HIV in the PBG (Fig. 2i). Six of the nine Hbonds connecting VL9 and HC in 1MHE are conserved in the RL9HIV complex. However, four novel bonds are formed, one of which (P9-Leu [OXT]-Lys-146 [NZ]) is also present in the Mtb44 complex. Glu-152, situated on the a2 helix, forms novel bonds with the P3-Tyr side chain and P7 backbone, the latter of which canonically bonds with Asn-77 on the $\alpha 1$ helix in 1MHE. Thus, P6 and 7 are stabilised closer to the a2 helix in RL9HIV than in other HLA-E-peptide complex structures.

Analysis of Mtb44 primary anchor residue variants. Peptides previously shown to bind HLA-E predominantly have Met at P2 for optimal B pocket binding, in addition to a strong $\mathrm{F}$ pocket binding preference for Leu at P9. We investigated these specificities using a single-chain peptide- $\beta 2 \mathrm{~m}-\mathrm{HC}$ construct, where $\mathrm{P} 2$ variants of the Mtb44 and VL9 peptides were tested for cellsurface expression in transfected 293T cells. As illustrated in Fig. 3a, the B pocket tolerated all hydrophobic side chains at P2, in addition to polar residues such as Gln, Ser and Thr. Despite Mtb44 and VL9 producing slightly varying hierarchies of binding for P2 substituted residues, cell surface expression was not supported by the charged residues Glu, Asp or Arg in either peptide. We then sought to analyse the ability of the $B$ pocket to accommodate a selection of these hydrophobic and polar residues, some of which are present in HLA-E restricted microbial peptides, by crystal structure determination of Mtb44 P2 variant peptide complexes. Diffraction quality crystals could be grown for HLA-E ${ }^{\star} 01: 03$ in complex with Mtb44 P2-Gln and Phe variants, termed Mtb44*P2-Gln (PDB ID: 6GH4) and Mtb44*P2-Phe (PDB ID: 6GGM), respectively. HLA-E*01:03 was also crystallised bound to an Mtb44 peptide in which the aliphatic hydrophobic P9 primary anchor, Leu, was substituted by the aromatic hydrophobic residue, Phe, termed Mtb44*P9-Phe (PDB ID: 6GHN).

Reflections from multiple isomorphous Mtb44* $\mathrm{P} 2$-Phe crystals were merged and integrated by Xia2, producing a dataset to $2.7 \AA$ in the P1 space group. Similarly, Mtb44 2 -Gln crystallised in P1, however, diffraction data were collected to a higher resolution of $2.1 \AA$ (Supplementary Table 1). Electron density was present for both peptides including the substituted P2 side chains (Fig. 3b).
Superposition of $\mathrm{Mtb} 44^{\star} \mathrm{P} 2$-Gln and $\mathrm{Mtb} 44^{\star} \mathrm{P} 2$-Phe with the original Mtb44 complex structure revealed similarly orientated peptides in the PBG (Fig. 3c). Perhaps unsurprisingly, considering its depth and hydrophobicity, P2 Leu to Gln or Phe substitutions were tolerated by the B pocket with minimal repositioning of the pocket-forming residues (Fig. 3d).

However, the P2 Mtb44*P2-Phe Ca atom is elevated $0.7 \AA$ away from the groove floor in the direction of the al helix, relative to Mtb44, permitting accommodation of the larger aromatic side chain (Fig. 3c). Additionally, P6 and 7 of the Mtb44P2-Phe variant sit 0.9 and $0.8 \AA$ deeper in their respective $\mathrm{C}$ and $\mathrm{E}$ pockets, in turn reducing the height of the P5 kink and increasing the P5 buried area $40 \%$ relative to Mtb44 and Mtb44*P2-Gln (Fig. 3e). Furthermore, Mtb44*P2-Phe forms three additional $\mathrm{H}$-bonds securing $\mathrm{P} 1$ and 2 in the groove. Such features align with single-chain trimer-based transfectant data demonstrating that Mtb44 $\mathrm{P} 2-\mathrm{Phe}$ drives the highest relative levels of HLA-E surface expression. However, these minor readjustments in peptide positioning due to $\mathrm{P} 2$ substitution do not disrupt immune recognition: $\mathrm{CD}^{+} \mathrm{T}$ cells isolated from the spleens of BCG-vaccinated RM mounted responses of similar magnitude when stimulated with Mtb44*P2-Phe, Mtb44*P2-Gln or the index Mtb44 epitope, emphasising the similarity in positioning of solvent exposed side chains and thus their antigenicity in vivo (Fig. 3f).

Reflections from multiple isomorphous Mtb44* ${ }^{*} 9$-Phe complex crystals were merged in Xia2, yielding a dataset in the P1 space group to $2.5 \AA$. Clear electron density was present for the peptide including the substituted P9-Phe side chain (Fig. 4a). Similarly to the Mtb44 P2 variants, Mtb44* ${ }^{\star} 9$-Phe adopts the classical kinked conformation in the PBG and exhibits minor repositioning relative to the original Mtb44 peptide (Fig. $4 \mathrm{~b}-\mathrm{d}$ (i) and (ii)). Superposition revealed a slight elevation in the P9 Ca atom of Mtb44 ${ }^{*} \mathrm{P} 9-\mathrm{Ph}$ compared to Mtb44 and an alternative rotamer for the F pocket-lining Phe-116 side chain, which tilts more acutely towards the groove floor, increasing pocket volume to accommodate the larger aromatic side chain (Fig. 4e). Minor repositioning also impacts the hydrogen-bonding network: three of the nine H-bonds securing Mtb44 to the groove are lost in Mtb44*P9-Phe at positions 1, 2 and 9.

Blue-native (BN)-PAGE gel signatures of HLA-E complexes. Despite the apparent homogenous nature of HLA-E refolded in the presence of RL9HIV, as indicated by size exclusion and ionexchange chromatography, it was not possible to obtain a reproducible thermal melt pattern for this complex in contrast to

Fig. 1 a Binding of RL9HIV and microbial peptides to human HLA-E. HLA-E $(1 \mu \mathrm{M})$ and $\beta 2 \mathrm{M}(1.5 \mu \mathrm{M})$ were refolded with RL9HIV/RL9SIV peptide (70 $\mu \mathrm{M})$ or with previously described HLA-E-restricted microbial epitopes. Following $48 \mathrm{~h}, \mathrm{HLA}-\mathrm{E}-\beta 2 \mathrm{M}$-peptide complex formation was assessed by sandwich ELISA. (i) Comparison of RL9HIV binding to VL9 (purple) and heavy chain (HC) $+\beta 2 \mathrm{M}$ only (dark grey) controls with peptide-binding cut-off noted (black dashed line). Data for five biological runs (two technical repeats/run) \pm the standard error of the mean (SEM) is reported. (ii) Comparison of RL9HIV binding to HLA-E restricted microbial epitopes (one biological run and two technical replicas \pm SEM). Colour coding as per (i). The $x$ axes denote peptide identity and the $y$ axes signify absorbance at $450 \mathrm{~nm}$. b RhCMV68-1-HIV vaccinated rhesus macaques (RM) develop Mamu-E-restricted responses to the RL9HIV epitope. Peripheral blood mononuclear cells (PBMCs) from six RhCMV68-1-HIVGag vector vaccinated RM were stimulated with the RL9HIV peptide in the presence of either control peptide, CLIP peptide + anti-MHC-II mAb (MHC-II-blocking) or VL9 peptide (MHC-E-blocking). CD8 ${ }^{+}$T cell recognition was determined by evaluating interferon $\gamma$ (IFN $\gamma$ ) and/or tumour necrosis factor (TNF $\alpha$ ) production by flow cytometric ICS assays. Negative control reflects identical incubations but without added peptide. Representative flow cytometric profiles with response frequencies of gated $\mathrm{CD}^{+}{ }^{,} \mathrm{CD}^{+}$ T cells in each quadrant and frequencies of responding cells (IFN $\gamma+$ and/or TNF $\alpha+$ ) in six vaccinated RM are reported. c Binding of Mycobacterium tuberculosis (Mtb)-derived peptides to human HLA-E. HLA-E $(1 \mu \mathrm{M})$ and $\beta 2 \mathrm{M}(1.5 \mu \mathrm{M})$ were refolded with Mtb-derived peptide (70 $\mu \mathrm{M})$. Following $48 \mathrm{~h}$, HLA-E- $\beta 2 M$-peptide complex formation was quantified by sandwich ELISA. VL9 positive and HC $+\beta 2 M$ only negative controls and the peptide-binding cutoff (black dashed line) are denoted. Data for three biological repeats (two technical replicas/repeat) with average absorbance \pm SEM are reported. Plot axes as described in (a). d BCG-vaccinated RM develop Mamu-E-restricted responses to the Mtb44 epitope. Peripheral blood mononuclear cells (PBMCs) from three BCG-vaccinated RM were stimulated with the Mtb44 peptide in the presence of either control peptide, CLIP peptide + anti-MHC-II mAb (MHC-II-blocking) or VL9 peptide (MHC-E-blocking). Analysis of $\mathrm{CD}^{+} \mathrm{T}$ cell recognition as described in (b) 
a

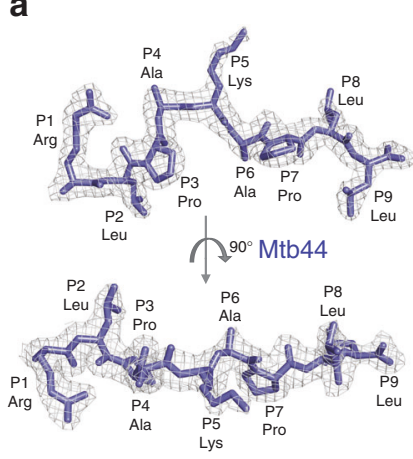

b

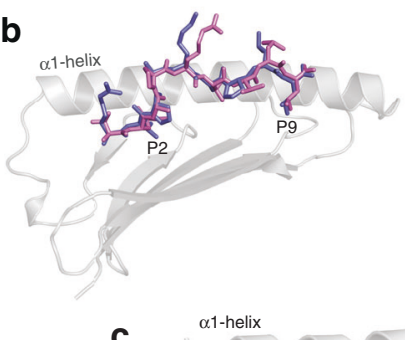

c

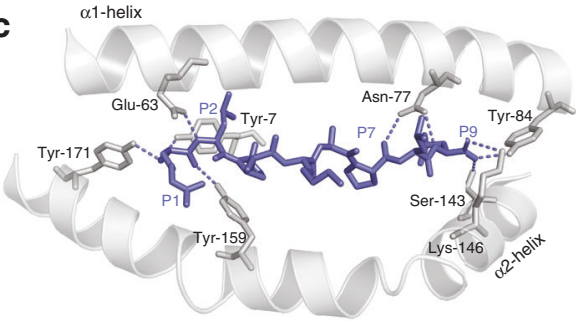

g

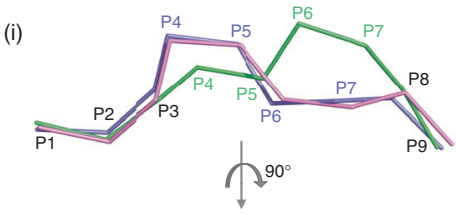

(ii)
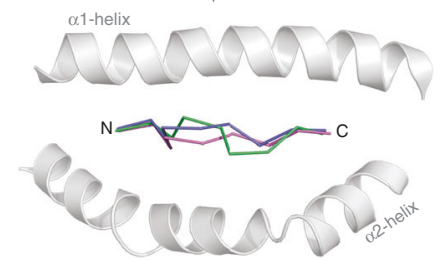

d

e

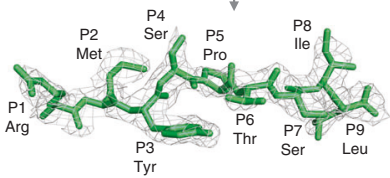

(ii)
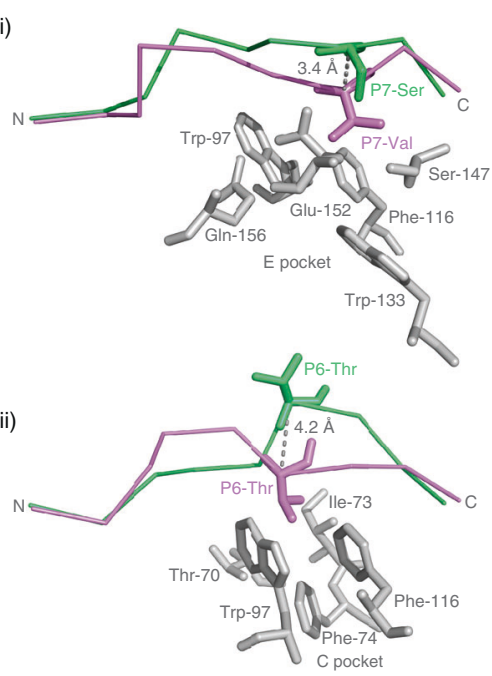

(iii)

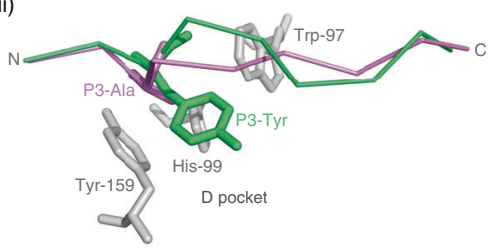

h
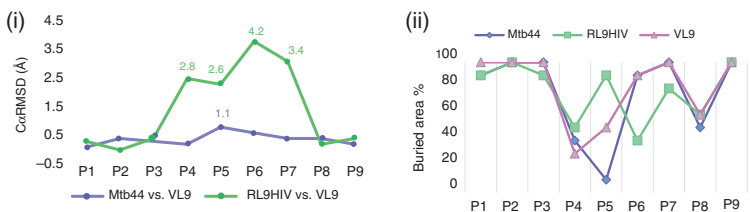

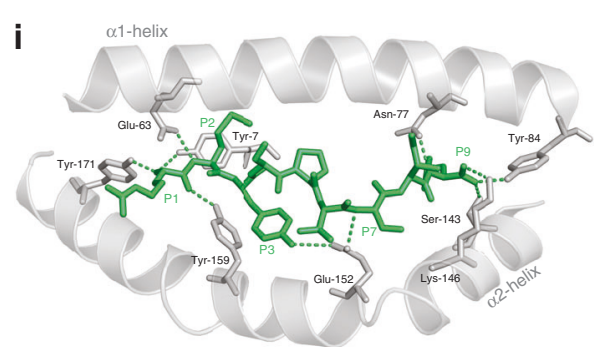

Fig. 2 Structural analysis of HIV- and Mtb-derived peptide-bound HLA-E*01:03 complexes. a Mtb-derived Mtb44 peptide (RLPAKAPLL) visualised as sticks in purple-slate side-on and from above with electron density overlaid in grey mesh and HLA-E*01:03 HC $+\beta 2 \mathrm{M}$ omitted for clarity. (All electron density contoured at 1 sigma.) b Alignment of Mtb44 (purple-slate) and canonical VL9 (VMAPRTVLL) (violet) peptides depicted as sticks in the peptide binding groove displayed in grey cartoon with the omission of the $\alpha-2$ helix for clarity. c Intermolecular Mtb44 peptide-HC hydrogen-bonding network visualisation. Peptide and HC-derived bonded residues displayed as solid sticks and H-bonds depicted as dashed lines (purple-slate). Peptide binding groove displayed as grey cartoon with the $\beta$-sheet floor omitted for clarity. d HIV-derived epitope "RL9HIV" (RMYSPTSIL) (lime green) in grey mesh electron density visualised side-on and from above with peptide binding groove omitted for clarity. e Alignment of RL9HIV (lime green) and canonical VL9 (violet) peptides depicted as sticks in the peptide binding groove with the $\alpha 1$ helix and $\beta$-sheet floor displayed in grey cartoon and the $\alpha 2$ helix omitted for clarity. $\mathbf{f}$ (i) $\mathrm{E}$, (ii) $\mathrm{C}$ and (iii) D pocket visualisation for RL9HIV (lime green) superposed to VL9 (violet) with pocket-forming residues derived from the heavy chain of the RL9HIV-HLA-E complex depicted as grey sticks. Distances between the superposed peptide $\mathrm{C} \alpha$ atoms shown as grey dashed lines. $\mathbf{g}$ C $\alpha$ backbone alignment of Mtb44, RL9HIV and VL9 peptides visualised side-on (i) and from above (ii) with peptide binding groove $\alpha 1+2$ helices depicted in grey cartoon. $\mathbf{h}$ (i) Distance in $\AA$ between superposed $\mathrm{C} \alpha$ atoms of Mtb44 versus VL9 and RL9HIV versus VL9 with peptide residue position along the $x$ axis and distance on the $y$ axis. (ii) Buried residue area percentage for Mtb44, RL9HIV and VL9 peptides with residue position along the $x$ axis and buried area \% on the $y$ axis. i Intermolecular RL9HIV peptide-HC hydrogen-bonding network visualisation. Peptide and HC-derived bonded residues displayed as solid sticks with $\mathrm{H}$-bonds depicted as dashed lines (lime green). Peptide binding groove displayed as grey cartoon with the $\beta$-sheet floor omitted for clarity 
VL9- and Mtb44-refolded HLA-E- $\beta 2 \mathrm{~m}$ material. The immediate incorporation of dye suggested issues relating to sample nonuniformity and stability, and indicated that heterogeneous protein species-either higher order aggregates or mixed protein formswere probably present. To explore this, we performed blue-native polyacrylamide gel electrophoresis (BN-PAGE) analysis to compare freshly purified RL9HIV-, Mtb44- and VL9-refolded HLA$\mathrm{E}-\beta 2 \mathrm{~m}$ complexes. Unusually, HLA-E and $\beta 2 \mathrm{M}$ readily formed dimers in the absence of exogenously added peptide, and this material was also included in these experiments. The results (Fig. 5a) illustrate distinct gel signatures for the various HLA-E$\beta 2 \mathrm{~m}$ samples. For HLA-E refolded in the presence of the higher affinity binding Mtb44 and VL9 peptides, singular, compactly formed bands ("compact form" (Cf)) resolved downstream of the $66 \mathrm{kDa}$ protein marker. The gel signatures of VL9- and Mtb44refolded HLA-E complexes were similar in form, but positionally distinct, presumably due to the charge difference of solvent exposed peptide residues (two positively charged amino acids for Mtb44 and one for VL9). In contrast, HLA-E-RL9HIV complexes resolved heterogeneously as two main bands: a faint, $\mathrm{Cf}$ that resolved comparably to the HLA-E-Mtb44 Cf species, and a more dominant, diffuse form (Df). Finally, HLA-E- $\beta 2 \mathrm{~m}$ refolded in the absence of peptide, resolved only as diffuse material similar to the Df observed for RL9HIV.

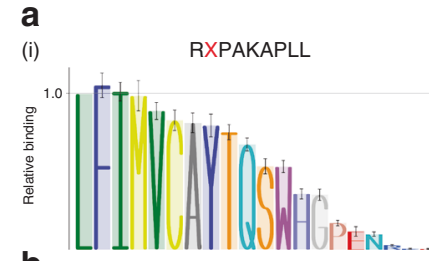

b

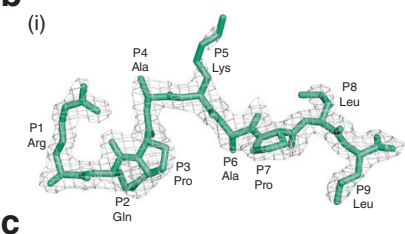

(i)
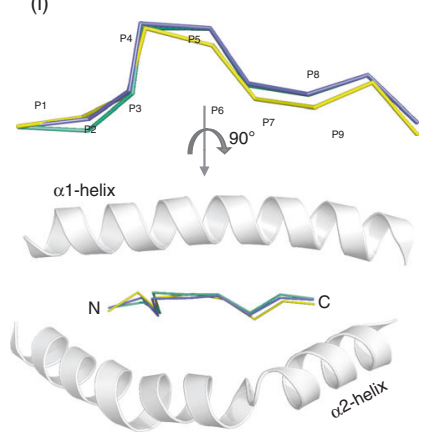

d

(i)

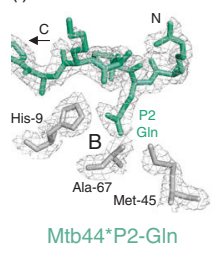

(ii)

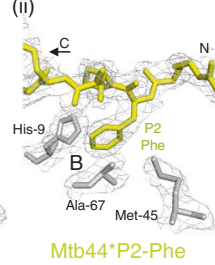

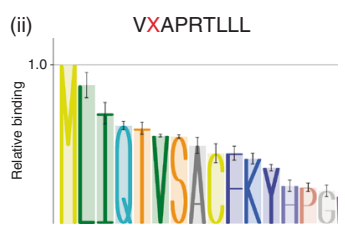

(ii)
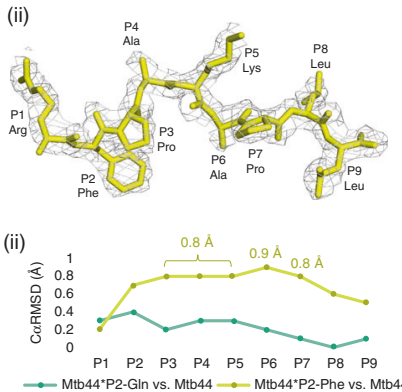

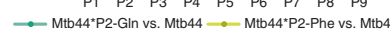

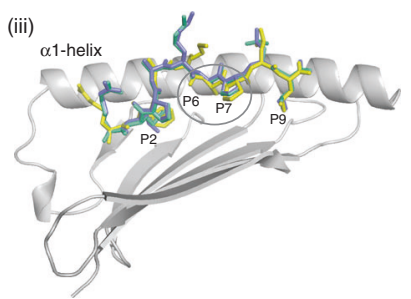

(iii)

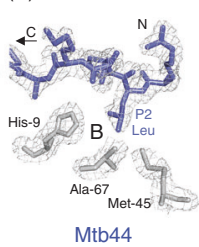

(iv)

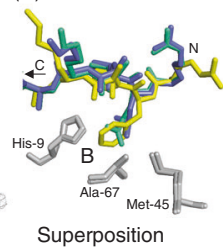

e

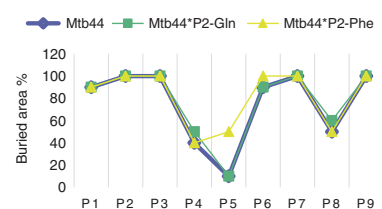

f
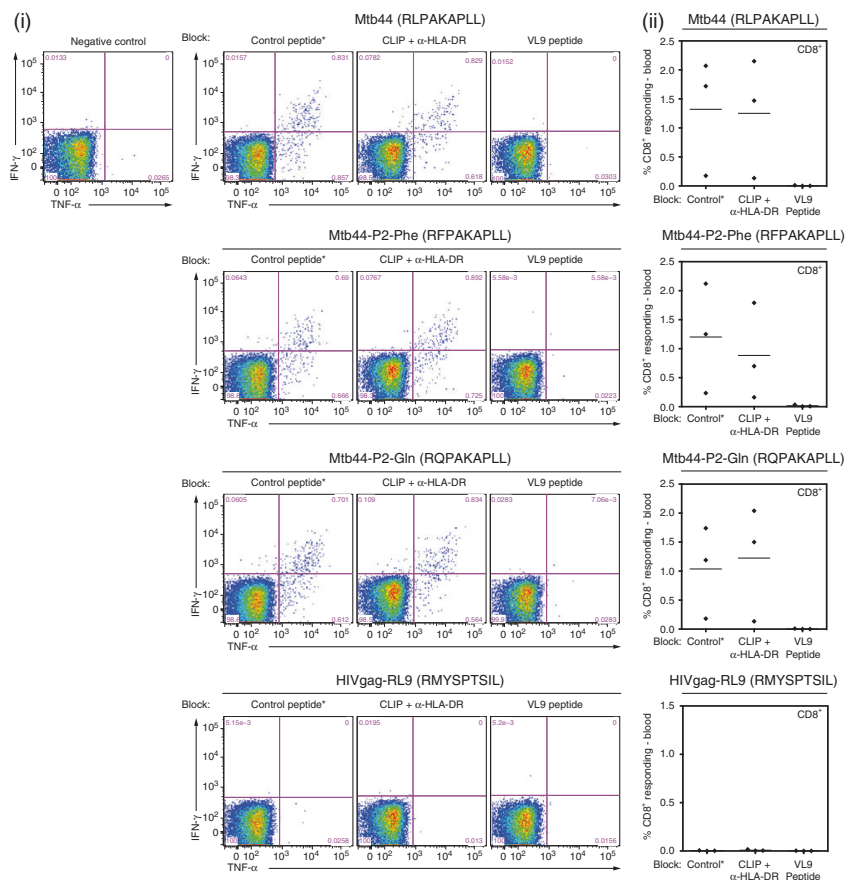

Fig. 3 Structural analyses of Mtb44 position 2 peptide variants: Mtb44*P2-GIn and Mtb44*P2-Phe. a Relative binding of Mtb44 (i) and VL9 (ii) position 2 peptide variants from single-chain peptide- $\beta 2 \mathrm{M}-\mathrm{HC}$ DNA constructs transfected in 293T cells and tested for HLA-E surface expression by flow cytometry using the MHC-E-specific 3D12 antibody. Data scaled relative to the index position 2 residue of each peptide (Leu for Mtb44 and Met for VL9). Relative binding displayed on the $y$ axis, with position 2 residue mutations on the $x$ axis. The mean \pm SEM of the MFI is reported $(n=4)$. $\mathbf{b}:(\mathbf{i})$ The Mtb44 position 2 Glutamine variant Mtb44*P2-GIn (green-cyan) and (ii) the Mtb44 position 2 phenylalanine variant Mtb44*P2-Phe (yellow) visualised side-on with electron density overlaid in grey mesh and $\mathrm{HC}+\beta 2 \mathrm{M}$ omitted for clarity. c (i) $\mathrm{C} \alpha$ backbone alignment of Mtb44*P2-Gln (green-cyan), Mtb44*P2-Phe (yellow) and Mtb44 (purple-slate) peptides visualised side-on and from above, respectively, with peptide binding groove $\alpha-1+2$ helices depicted in grey cartoon. (ii) Distance in $\AA$ between superposed $\mathrm{C} \alpha$ atoms of Mtb44*P2-GIn versus Mtb44 and Mtb44*P2-Phe versus Mtb44 with peptide residue position along the $x$ axis and distance $(\AA)$ on the $y$ axis. (iii) Superposition of Mtb44*P2-GIn (green-cyan), Mtb44*P2-Phe (yellow) and Mtb44 (purple-slate) peptides with peptide binding groove $\alpha 1$ helix and $\beta$-sheet floor depicted in grey cartoon. $\mathbf{d}$ B pocket visualisation for (i) Mtb44*P2-GIn (ii) Mtb44*P2-Phe (iii) Mtb44 and (iv) Mtb44*P2-Gln/Mtb44*P2-Phe/Mtb44 superposition. HLA-E*01:03 HC-derived pocket-forming residues depicted as grey sticks with electron density overlay as grey mesh. e Buried residue area percentage for Mtb44^P2-Phe, Mtb44*P2-Gln and Mtb44 peptides with residue position along the $x$ axis and buried area \% on the $y$ axis. f BCG-elicited Mamu-E-restricted Mtb44-specific CD8 ${ }^{+}$T cells recognise Mtb44 position 2 variants. Peripheral blood mononuclear cells (PBMCs) from 3 BCG-vaccinated RM were stimulated with the Mtb44 (RLPAKAPLL) peptide, the position 2 peptide variants $\left[M t b 44^{\star}\right.$ P2-Phe (RFPAKAPLL), Mtb44*P2-GIn (RQPAKAPLL)] and the HIVgag-derived RL9HIV peptide. CD8 ${ }^{+} \mathrm{T}$ cell recognition determined and shown as described in Fig. $1 \mathrm{~b}$ 
To determine if these gel signatures are comparable to classical MHC class I, the BN-PAGE gel profiles of HLA-B ${ }^{\star} 57-\beta 2 \mathrm{~m}$ complexes refolded with epitope peptide were also explored. As depicted in Fig. 5b, Cf signatures exclusively represented all samples tested, confirming that the compact gel signature species most likely represents optimally folded, peptide-loaded MHC class I protein.

We next assessed whether the HLA-E Cf and Df species represented HLA-E- $\beta 2 \mathrm{~m}$ co-complexes, as indicated by the presence of both the $\mathrm{HC}$ and $\beta 2 \mathrm{~m}$. This was particularly
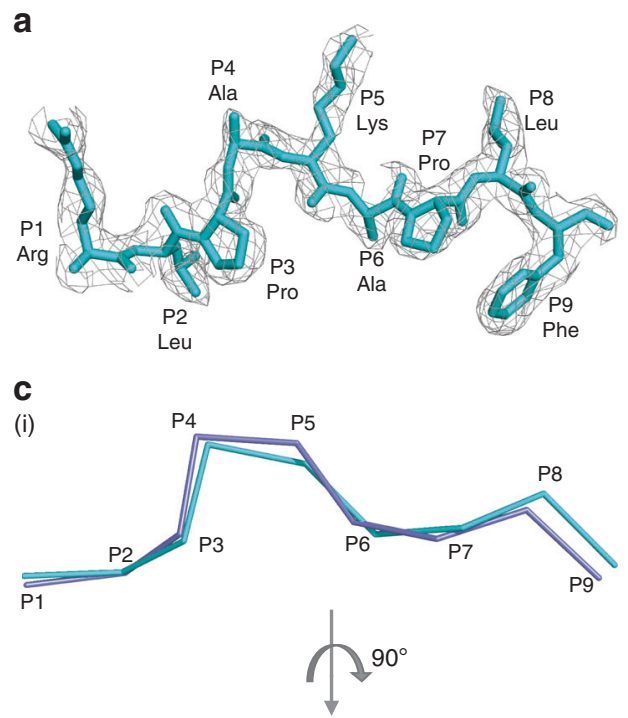

(ii)
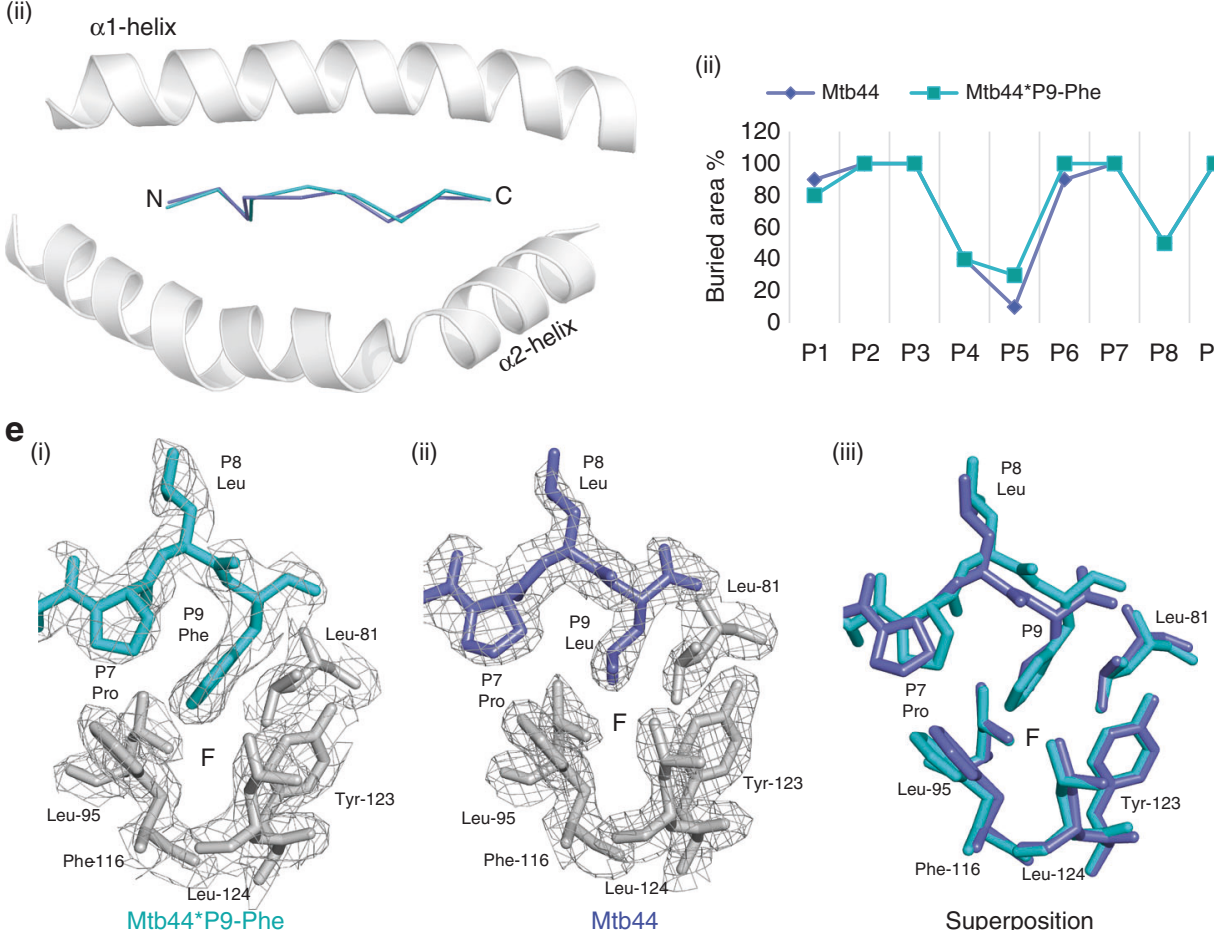

Fig. 4 Structural analyses of the Mtb44 position 9 peptide variant: Mtb44*P9-Phe. a The Mtb44 position 9 phenylalanine variant Mtb44*P9-Phe (bluecyan) visualised side-on with electron density overlaid in grey mesh and HLA-E*01:03 HC $+\beta 2 \mathrm{M}$ omitted for clarity. b Superposition of Mtb44*P9-Phe (blue-cyan) and Mtb44 (purple-slate) peptides depicted as sticks with the binding groove $\alpha 1$ helix and $\beta$-sheet floor depicted in grey cartoon. c C $\alpha$ backbone alignment of Mtb44*P9-Phe (blue-cyan) and Mtb44 (purple-slate) peptides visualised side-on (i) and from above (ii), with binding groove $\alpha 1+$ 2 helices depicted as grey cartoon. $\mathbf{d}$ (i) Distance in $\AA$ between superposed $C \alpha$ atoms of Mtb44*P9-Phe versus Mtb44 with peptide residue position on the $x$ axis and distance $(\AA)$ on the $y$ axis. (ii) Buried residue area percentage for Mtb44*P9-Phe (blue-cyan) and Mtb44 (purple-slate) peptides with residue position along the $x$ axis and buried area \% on the $y$ axis. e F pocket visualisation of (i) Mtb44*P9-Phe (ii) Mtb44 and (iii) Mtb44*P9-Phe/ Mtb44 superposed complexes. HLA-E*01:03 HC-derived F pocket-forming residues depicted as grey sticks with electron density overlaid as grey mesh 
a

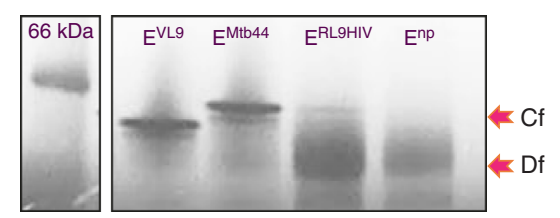

b

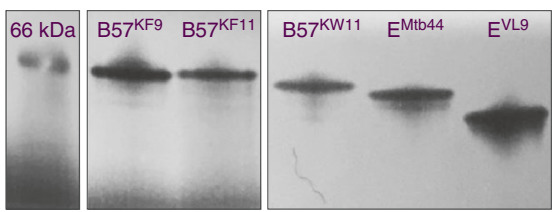

C

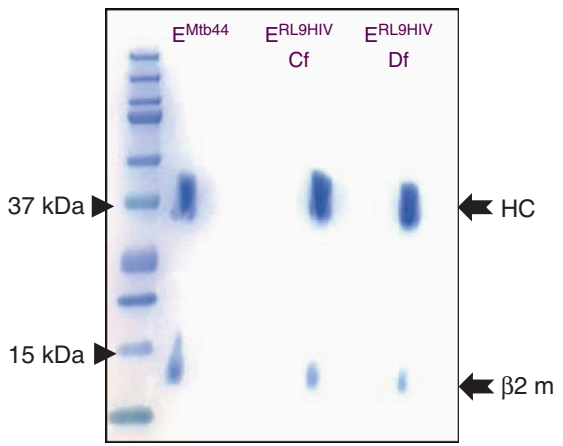

d

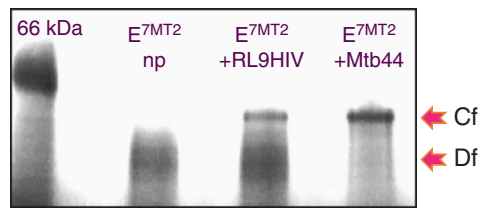

e

(i)

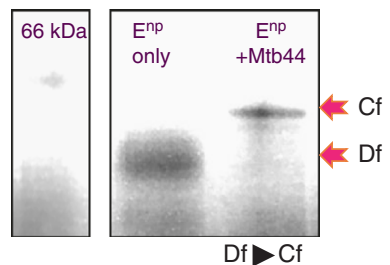

(ii)

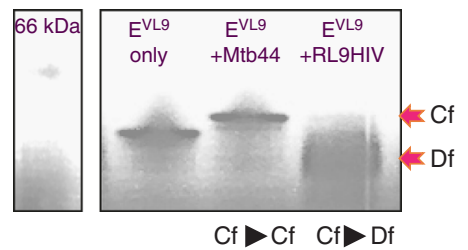

Fig. 5 Blue-native-PAGE analysis of HLA-E complexes. a Blue-native (BN) gel signature of refolded HLA-E complexes. Purified HLA-E- $\beta 2 m$ complexes refolded without peptide (no peptide (Enp)) or in the presence of RL9HIV (ERL9HIV), Mtb44 (EMtb44) or VL9 (EVL9) peptides were visualised by BN-PAGE gel. Red arrows indicate protein gel forms comprising compact (Cf) and diffuse (Df) species. The $66 \mathrm{kDa}$ protein marker is highlighted for reference. b HLA-E compact (Cf) gel signatures resembled MHC class la folded proteins. Purified HLA-B57- $32 \mathrm{~m}$ complexes refolded in the presence of the previously defined KAFSPEVIPMF (B57 KF11), KAAFDLSFF (B57 KF9) and KAYDTEVHNVW (B57 KW11) epitopes were visualised by blue-native (BN) PAGE gel for comparison to HLA-E complexes refolded with VL9 and Mtb44. The $66 \mathrm{kDa}$ protein marker is noted for reference. c 2D analysis of BN-gel resolved HLA-Epeptide refolds. BN-gel separated HLA-E-RL9HIV and HLA-E-Mtb44 complexes were 2D resolved via NuPAGE 10\% Bis-Tris using standard 12-well lane gels where individual $\mathrm{BN}$-gel slices were excised and individually separated. Heavy chain (HC) and $\beta 2 \mathrm{~m}$ bands are noted (black arrows), and reference protein size markers are displayed (arrowheads). d BN-gel signatures of UV peptide labile-HLA-E complexes (E7MT2) following RL9HIV, Mtb44 and no peptide rescue. Pre-purified HLA-E molecules in complex with UV-labile 7MT2 were illuminated without peptide (np) or in the presence of RL9HIV and Mtb44 epitopes. The material was subsequently resolved by BN-gel. Red arrows indicate protein gel forms. The $66 \mathrm{kDa}$ protein marker is noted for reference. e Exogenous peptide loading into no peptide and VL9-refolded HLA-E complexes. Purified HLA-E- $\beta 2 \mathrm{~m}$ complexes refolded (i) without peptide $\left(E^{n p}\right)$ were pulsed with 50 M excess Mtb44 peptide (ii) and pre-refolded HLA-E VL9 peptide complexes (EVL9) were pulsed with 200 M excess Mtb44 or RL9HIV peptides after which BN-gel signatures were analysed. Gel signature transitions are noted (text beneath images). Abbreviations throughout: Cf compact form; $D f$ diffuse form

We questioned whether pre-refolded HLA-E- $\beta 2 \mathrm{~m}$, with optimal tertiary structure and disulphide-bridge formation, was more receptive to RL9HIV peptide binding. To evaluate this, the method of UV-mediated peptide exchange was employed ${ }^{29}$. HLA-E refolded with the UV-labile VL9-based 7MT2 epitope incorporating a light sensitive $J$ moiety at position 5 along the peptide was photo-illuminated in the presence or absence of 100 $\mathrm{M}$ excess Mtb44 and RL9HIV "rescue" peptides, and subsequently evaluated by BN-gel analysis. In accordance with observations made for conventionally refolded HLA-E complexes, Mtb44-exchanged material had a clear Cf signature whereas the RL9HIV fraction retrieved following peptide exchange comprised both diffuse and compact gel forms (Fig. 5d).

To assess whether HLA-E- $\beta 2 \mathrm{~m}$ complexes, previously refolded in the absence of peptide, were peptide-receptive, their BN-gel signatures were evaluated following incubation with $50 \mathrm{M}$ excess of Mtb44 peptide. As indicated by the transition of gel signatures from Df to Cf species (Fig. 5e(i)), peptide-receptivity was also a feature of the presumed peptide "empty" HLA-E- $\beta 2 \mathrm{~m}$ forms.

The ability of HLA-E- $\beta 2 \mathrm{~m}$ peptide-loaded material to exchange peptide was also evaluated. As Mtb44 and VL9refolded HLA-E- $\beta 2 \mathrm{~m}$ complexes produced BN-PAGE Cf gel signatures that are position-distinct, this feature was used as a tool to gauge peptide exchange. Previously refolded and purified HLA-E- $\beta 2 \mathrm{~m}$-VL9 peptide complexes were incubated with $200 \mathrm{M}$ excess of Mtb44 peptide and subsequently analysed by BN-PAGE. As evidenced by the VL9 to Mtb44 Cf gel signature transition, VL9-loaded HLA-E complexes were readily displaced by the Mtb44 peptide (Fig. 5e(ii)). Finally, we tested whether the pre-refolded HLA-E- $\beta 2 \mathrm{~m}$-VL9 peptide complexes were also susceptible to RL9HIV peptide exchange. In agreement with observations made for the higher affinity Mtb44 peptide, $200 \mathrm{M}$ excess of RL9HIV also displaced VL9 from HLA-E- $\beta 2 \mathrm{~m}$ complexes, as evidenced by the emergence of gel forms 
resembling the HLA-E- $\beta 2 \mathrm{~m}$-RL9HIV pre-refolded complex signature (Fig. 5e(ii)).

\section{Discussion}

Mamu-E restricted $\mathrm{CD}^{+} \mathrm{T}$ cell responses have been implicated as immune correlates of protection in RhCMV68-1-vectored SIV vaccination trials, triggering new interest in HLA-E as a potential driver of protective immunity against HIV-1 $13,30-32$. Although earlier work indicated that the HLA-E binding repertoire was restricted to MHC class Ia leader sequence-derived peptides for presentation to NK cells, it is increasingly apparent that HLA-E can also bind and present autologous and microbially derived peptides to $\mathrm{CD}^{+} \mathrm{T}^{-}$cells ${ }^{3,15,18-20,33-36}$. The breadth of epitopes identified in RhCMV68-1 SIV vaccine studies and mycobacterial infection ${ }^{15}$ indicates that both Mamu-E- and HLA-E-restricted peptide repertoires have the potential to be very diverse ${ }^{13}$. In particular, the range of Mamu-E restricted RhCMV68-1 stimulated responses is consistent with increased diversity of HLA-E peptide binding in transporter-associated with antigen processing (TAP)-deficient cell lines ${ }^{37}$. Despite sequence disparity between the human and rhesus homologues, the amino acids that comprise the five primary and secondary anchor residueaccommodating pockets are almost identical between HLA-E and 21 of the 22 Mamu-E alleles, with a single exception, I73T, in the $\mathrm{C}$ pocket (Supplementary Table 3). Furthermore, it has previously been shown that rhesus SIV-specific $\mathrm{CD}^{+} \mathrm{T}$ cells recognise peptides presented by both Mamu-E and HLA-E ${ }^{13}$. Similarly, rhesus $\mathrm{CD} 8^{+} \mathrm{T}$ cell responses to RL9HIV, Mtb44 or Mtb44 P2 variant epitopes were of comparable magnitude when peptides were presented by either Mamu-E or HLA-E (Supplementary Figs. 2, 3), further supporting the functional similarity between the rhesus and human homologues ${ }^{38}$. Finally, in a singlechain peptide- $\beta 2 \mathrm{M}-\mathrm{HC}$ trimer-based comparison of VL9 P2 variant binding to $\mathrm{HLA}-\mathrm{E}^{\star} 01: 03$ versus $\mathrm{Mamu}-\mathrm{E}^{\star} 02: 04$, the human and rhesus homologues exhibited similar binding preferences (Supplementary Fig. 1A). Thus, although there are no published structures of Mamu-E, it is reasonable to extrapolate from these experimental data and the close relatedness of HLA-E that the two homologues behave similarly in folding and lowaffinity peptide binding.

The breadth of the HLA-E peptidome was re-explored using a refolding-ELISA approach that quantified the relative capacity of peptides to stabilise HLA-E- $\beta 2$ M-peptide complex formation ${ }^{8,39}$ (Fig. 1a, c). Several HIV epitopes identified in RhCMV68-1 HIV1 Gag-insert vaccine trials, including some that lacked canonical anchor residues, exhibited reproducible binding to HLA-E, albeit with considerably lower affinity than VL9 peptides (L. Picker, unpublished). Similarly, screens of previously reported HLA-E restricted microbial peptides, including an Mtb-derived panel, highlighted a selection that supported heterotrimeric complex formation including one, Mtb44, which exhibited comparable binding affinity to VL9 peptides. This assay also indicated that HLA-E and $\beta 2 \mathrm{~m}$-fold in the absence of added peptide, as evidenced by reproducible signals for peptide-free refolded samples.

As previous structures of HLA-E were crystallised in complex with highly conserved, canonical VL9 peptides or HCMV UL40 protein-derived VL9 mimics, it was unclear how nonleader sequence epitopes could be accommodated in the PBG. To understand the structural basis of binding, two of the highest affinity pathogen-derived peptides identified in refolding-ELISAs, RL9HIV (HIV gag) and Mtb44 (Mtb), were selected for crystallographic analysis (Figs. 1, 2). Irrespective of sequence disparity to VL9, including a positive charge at P1 and a primary anchor Met to Leu substitution at P2, Mtb44 exhibited strong conformational similarity, adopting the classical kinked binding motif in the PBG.
However, despite a similar backbone conformation and solvent exposure profile to VL9, Mtb44 possesses sufficiently distinct exposed side chains to elicit specific, Mamu-E restricted $\mathrm{CD} 8^{+} \mathrm{T}$ cell responses in BCG-vaccinated macaques. In contrast, the RL9HIV peptide carries canonical anchors Met at P2 and Leu at $\mathrm{P} 9$, yet adopts an alternative C-terminally shifted kinked motif in the binding groove, resulting in a distinct solvent exposed signature with implications for immunogenicity and peptide-specific TCR interaction. This alternative backbone conformation, in turn, disrupts secondary $\mathrm{C}$ and $\mathrm{E}$ pocket occupancy with the P6 and 7 anchors projecting 4.2 and $3.4 \AA$ further into the solvent, respectively, likely contributing to the lower overall stability of the complex.

In line with diverse and non-canonical SIVGag epitopes defined by Hansen et al. ${ }^{13}$, we provide structural evidence illustrating that the $\mathrm{B}$ and $\mathrm{F}$ pockets of HLA-E can tolerate a wider range of side chains than previously predicted (Figs. 3, 4). HLA-E structures crystallised in complex with Mtb44 variants encoding P2-Gln or Phe, or P9-Phe, demonstrated minimal conformational repositioning relative to Mtb44 in the PBG. However, these primary anchor mutations did have minor ramifications on complex stability, via the gain (for Phe at position 2) or loss (for the Phe position 9 variant) of three H-bonds. Notably, Mamu-E restricted Mtb44-specific $\mathrm{CD}^{+} \mathrm{T}$ cell responses in BCG-vaccinated macaques were preserved when the animals were challenged with mutant peptides harbouring Phe or Gln at P2. This supports the possibility of enhancing MHC-epitope complex stability via primary anchor optimisation of certain HLA-E-restricted epitopes in immunogen design without disrupting TCR recognition $^{40}$. A more comprehensive analysis of B pocket tolerability was conducted by examining surface expression of single-chain peptide- $32 \mathrm{M}-\mathrm{HC}$ trimers encoding Mtb44 P2 variants. The hierarchy of tolerated residues is consistent with our structural data, with P2-Phe up-regulating surface expression of HLA-E to the greatest degree. In fact, this assay indicates that the B pocket is capable of accommodating any hydrophobic residue, dramatically increasing the potential number of HLA-E restricted HIV-derived vaccine candidate epitopes. The single-chain-trimer data also indicate that tolerance of primary anchor substitution is to some extent dependent on the remaining amino acids that constitute the nonameric peptide, as when P2 substitutions in the Mtb44 and VL9 peptides were compared, HLA-E surface expression was up-regulated in different ranking orders.

During the biochemical characterisation of HLA-E, we noted that, unusually, HLA-E HC and $\beta 2 \mathrm{~m}$ assembled as heterogeneous dimers in the absence of added peptide (Fig. 5). These dimeric forms likely comprised transition-state intermediates as evidenced by their diffuse gel profiles and the multiple transition states observed during thermal melt analysis. It is possible that peptide fragments derived from partially degraded HLA-E HC or $\beta 2 \mathrm{M}$ are present in the binding groove of these refolded species and it has previously been shown that peptide fragments as short as two amino acids ("dipeptides") are capable of stabilising the F pocket ${ }^{41}$. However, it is not critical whether these refolded species are truly empty. More importantly, peptide-receptivity is a characteristic of these "empty" HLA-E- $\beta 2 \mathrm{~m}$ forms, a finding that also concurs with data generated using particular peptide-free MHC class Ia- $\beta 2 \mathrm{~m}$ heterodimers ${ }^{42-44}$. Intrinsic allotype-specific differences in the propensity of MHC folding intermediates to retain peptide-receptivity exist, presumably reflecting the extent to which different allelic forms maintain these stable functional states. In relation to PBG integrity in the absence of peptide, recent Molecular Dynamic Simulation studies predict that the a1- $\alpha 2$ helices of HLA-E are rigid and remain open when devoid of peptide, which contrasts with classical molecules such as HLAA2 whose malleable helices are projected to collapse without 
peptide $^{13}$. This, in addition to the apparent stability of the HLAE- $\beta 2 \mathrm{~m}$ peptide-free heterogeneous form identified here, may provide insight into the features that contribute to the broad peptide receptivity of HLA-E and Mamu-E where classical TAPdependent peptide loading is blocked, for example, by various CMV genes and when RhCMV68-1 tropism is restricted to as yet undefined cell types in vivo ${ }^{13}$.

During our biochemical evaluation we also observed that HLAE-bound VL9 leader peptide was readily displaced by exogenous challenge with Molar excess of the Mtb44 peptide. Additionally, an excess of lower affinity peptides appears to disrupt the integrity of VL9-refolded HLA-E complexes, as evidenced by the emergence of the "diffuse" dimer gel form upon challenge with the RL9HIV epitope. Remarkably, the highest affinity VL9 leader epitope imparts a relatively small increase $\left(\mathrm{Tm} \leq 10^{\circ} \mathrm{C}\right)$ to the stability of the HLA-E- $\beta 2 \mathrm{~m}$ complex versus no peptide refolded forms, which contrasts the much larger values that high affinity peptides contribute to MHC class Ia stability upon binding ${ }^{8,10}$. Presumably this property of lower peptide affinity is driven from the perspective of $\mathrm{NK}$ cell recognition ${ }^{45,46}$. However, in the context-specific setting generated by the RhCMV68-1 regimen, this feature might enhance peptide-exchange properties of rerouted HLA-E- $\beta 2 \mathrm{~m}$ complexes.

Based on the gel profiles of optimally loaded peptide complexes, it is highly likely that the homogeneous, Cf of HLA-ERL9HIV represented material that crystallised in vitro. In contrast, the diffuse, peptide-receptive RL9HIV-specific material may comprise suboptimally bound peptide forms. The apparent incomplete loading of RL9HIV and other vaccine-identified peptides presumably reflects their lower affinities, given that exogenous loading of the higher affinity VL9 and Mtb44 peptides facilitated complete recovery of peptide-loaded, compact forms. It was initially reported that the MHC PBG undergoes conformational readjustments upon peptide binding ${ }^{47}$, with more recent hypotheses purporting that this is characterised by a two-stage transition where the groove initially exists in an "open" partially hydrated form accommodating suboptimally bound peptide, that converts ultimately, to a dehydrated, "closed" form upon optimal peptide loading ${ }^{48,49}$. A likely explanation is that the diffuse forms described here could include both "empty" heterodimer (open (o)) and weakly bound RL9HIV peptide (open-peptide (op)), of which the latter species transitions to the compact peptide bound form $(\mathrm{Cp})$ at an equilibrium primarily influenced by the affinity of the epitope for HLA-E (Hßo $\Leftrightarrow H \beta o p \Leftrightarrow H \beta c p)$. Thus, for the higher affinity VL9 and Mtb44 peptides, the balance is strongly skewed to the compact peptide-bound forms, whereas for weaker epitopes such as RL9HIV, the equilibrium is shifted towards the open/open-peptide binding species. In Mtb and RhCMV68-1 infected cells we suggest that movement of peptide binding away from the quality control environment of the ER-based TAPTapasin-associated PLC to an alternative loading pathway potentially shifts the balance allowing low-affinity peptides with suboptimal sequence motifs (op forms) to bind HLA-E or MamuE. Whether MHC peptide editors such as TAP-binding protein related (TAPBPR) protein, further influence peptide selection in this alternative pathway is currently unknown ${ }^{50-53}$. The work of Hansen et al. ${ }^{13}$ suggests that RhCMV68-1 vaccinated macaques prime Mamu-E-restricted $\mathrm{CD}^{+} \mathrm{T}$ cells that subsequently recognise peptide targets on SIV infected cells following viral challenge ${ }^{54}$. The mechanism underlying how these low-affinity peptide epitopes elicit $\mathrm{CD}^{+}{ }^{+} \mathrm{T}$ cell responses remains unclear. It is also unknown whether unusual forms of Mamu-E resembling the HLA-E 'open' material described here are generated in RhCMV68-1 vaccinated macaques, and whether $\mathrm{CD}^{+} \mathrm{T}$ cells recognise these forms. Both the mechanisms underlying alternative presentation routes and the features of peptide recognition by Mamu-E restricted $\mathrm{CD} 8^{+} \mathrm{T}$ cells are the focus of ongoing investigations.

\section{Methods}

Peptide synthesis. Synthetic nonameric peptides were generated by Fmoc (9fluorenylmethoxy carbonyl) chemistry to a purity of $85 \%$ by Genscript USA. All peptides were provided as lyophilised power, reconstituted in DMSO to a concentration of $200 \mathrm{mM}$, and stored at $-80^{\circ} \mathrm{C}$. A UV photolabile version of the HLAB leader sequence peptide, VMAPRTLVL, incorporating a UV-sensitive 3-amino3-(2-nitrophenyl)-propionic acid residue (J residue) substitution at position 5 (termed 7MT2), was synthesised by Dris Elatmioui at LUMC, The Netherlands. The 7MT2 peptide was stored as lyophilised power, and dispensed/reconstituted as required.

RM and vaccines. A total of 9 purpose-bred male RM (Macaca mulatta) of Indian genetic background (3-7 years of age) were used in the animal experiments reported here. All animals were used with the approval of the Oregon National Primate Research Center (ONPRC) Institutional Animal Care and Use Committee, under the standards of the US National Institutes of Health Guide for the Care and Use of Laboratory Animals (IACUC). The ONPRC is accredited as a Category 1 facility by the American Association for Accreditation of Laboratory Animal Care (AAALAC) and has an approved Assurance (\#A3304-01) for the care and use of animals on file with the Office for Protection from Research Risks at NIH. The IACUC adheres to national guidelines outlined in the Animal Welfare Act (7 U.S.C. Sections 2131-2159) and the Guide for the Care and Use of Laboratory Animals57 as mandated by the US Public Health Service Policy. The ONPRC IACUC approved care of RM, in addition to all experimental protocols and procedures. All RM were housed at the ONPRC in animal biosafety level (ABSL)-2 with autonomously controlled lighting, temperature and humidity. They were fed with commercially prepared primate chow (Purina Lab Diet: Fibre-Balanced Monkey Jumbo, 5000; High Protein Monkey Diet, 5045) twice daily and received daily supplemental fresh fruit or vegetables. Fresh, potable water was provided via automatic water systems. Physical exams, including body weight and complete blood counts, were performed at all protocol time points. A number of criteria were used to normalise the animals within vaccine groups, including MHC haplotype, age and sex. Once the groups were set, compatible animals within the same vaccine group were pair housed for the duration of the immunisation phase. Animals for which no compatible pair mate was identified, but otherwise met the pairing criteria, were single cage-housed for the duration of the immunisation phase or until a suitable compatible pair mate were identified. RM used in these experiments were free of cercopithicine herpesvirus 1, D-type simian retrovirus, and simian Tlymphotrophic virus type 1. Six RM (3-4 years of age) were subcutaneously vaccinated with the Rhesus cytomegalovirus 68-1 strain (RhCMV 68-1) (11 $^{2}$ expressing HIV-M-Gag-Nef fusion and HIV-M-Pol from the Episensus1 (RL9 RMYSPVSIL) or Episensus1 and Episensus2 (RL9 RMYSPTSIL) ${ }^{55}$. Three RM were immunised intravenously with $12.5 \times 10^{6}-1 \times 10^{8}$ colony forming units (cfu) of TICE ${ }^{\oplus}$ Strain Bacillus Calmette-Guérin (BCG) re-suspended in $3 \mathrm{~mL}$ of preservative-free saline.

Generation of Mamu-E`02:04 transfectant. The Mamu- $E^{\star} 02: 04$ transfectant was generated as previously described via ligation of the allele into the pCEP4 plasmid, sequence confirmation of the gene insert, and then electroporation of the Mamu-E*02:04 pCEP4 plasmid using Nucleofector II/Kit C (Lonza) into the MHCI null cell line $\mathrm{K} 562^{13,40}$. Mamu-E transfectants were maintained on drug selection (Hygromycin B) and routinely confirmed for surface expression of MHC-I by staining with pan-MHC-I antibody clone W6/32. The HLA-E ${ }^{\star} 01: 03$ transfectant was generously provided by Thorobald van Hall. In order to stabilise HLA-E and Mamu-E surface expression, transfectants were incubated at $27^{\circ} \mathrm{C}$ for $>3 \mathrm{~h}$ prior to use in assays and maintained at $27^{\circ} \mathrm{C}$ throughout peptide incubation until combined with effectors used in assays and maintained at $27^{\circ} \mathrm{C}$ throughout peptide incubation until combined with $\mathrm{CD}^{+}$effector cells. Surface MHC-E expression was confirmed via staining with W6/32 prior to use in $\mathrm{T}$ cell presentation assays.

T cell assays. HIV- and Mtb-specific $\mathrm{CD} 8^{+} \mathrm{T}$ cell responses were measured in mononuclear cell preparations from blood by flow cytometric ICS, as previously described in detail ${ }^{13,31}$. Briefly, mononuclear cells were incubated with peptide and the costimulatory anti-CD28 (CD28.2: Purified; $500 \mathrm{ng} / 1 \mathrm{e} 6$ cells; eBioscience, 7014-0289-M050) and anti-CD49d mAbs (9F10: Purified; 500 ng/1e6 cells; eBioscience, 7014-0499-M050) for $1 \mathrm{~h}$, followed by addition of $5 \mu \mathrm{g} / \mathrm{mL}$ Brefeldin A (BioLegend, 420601) for an additional $8 \mathrm{~h}$. Costimulation without antigen served as a background control (no stim). MHC restriction of the response was determined by pre-incubating isolated mononuclear cells for $1 \mathrm{~h}$ at room temperature in the absence or presence of a control peptide (SIVgag-CM9 CTPYDINQM; $20 \mu \mathrm{M}$ ), anti-MHC-II block (anti-HLA-DR mAb; clone-G46.6; $10 \mu \mathrm{g} / \mathrm{mL}$; BD Biosciences, 556642 ) and CLIP peptide block (MHC-II-associated invariant chain, amino acids 89-100; $20 \mu \mathrm{M}$ ), or the Mamu-E blocking peptide VL9 (VMAPRTLLL; $20 \mu \mathrm{M}$ ) prior to addition of test peptides. Stimulated cells were fixed, permeabilised and stained prior to flow cytometric analysis using an LSR-II instrument (BD Biosciences). Analysis was done using FlowJo software (Tree Star). In all analyses, progressive gating on the $\mathrm{CD} 3^{+}$population, and then the $\mathrm{CD} 4^{+} / \mathrm{CD} 8^{-}$versus 
$\mathrm{CD}^{-} / \mathrm{CD}^{+} \mathrm{T}$ cell subsets followed gating on the light scatter signature of small lymphocytes. Antigen specific $\mathrm{CD} 8^{+} \mathrm{T}$ cell response frequencies were determined from intracellular expression of IFN- $\gamma$ and TNF- $\alpha$. Boolean gates of (CD69+/TNF$\alpha^{+}$and/or $\mathrm{CD}^{+} 9^{+} / \mathrm{IFN}-\gamma^{+}$) were determined on the gated (responding) CD8 $+\mathrm{T}$ cell population ${ }^{56}$. The following conjugated antibodies were used in these flow cytometric ICS analyses: anti-CD4 (L200: BV510; $10 \mathrm{ng} / 1 \mathrm{e} 6$ cells; BD Biosciences, 563094), anti-CD3 (SP34-2: Pacific Blue; 150 ng/1e6 cells; BD Biosciences, 558124), anti-CD8a (SK1: PerCP-eFluor 710; 7.5 ng/1e6 cells; eBioscience, 46-0087-42), anti-IFN- $\gamma$ (B27: APC; $100 \mathrm{ng} / \mathrm{le} 6$ cells; BD Biosciences, 624078), anti-TNF- $\alpha$ (MAB11: FITC; 180 ng/test; BD Biosciences, 624046 and PE; 180 ng/1e6 cells; BD Biosciences, 624049) and anti-CD69 (FN50: PE; $30 \mathrm{ng} / \mathrm{le} 6$ cells; eBioscience, CUST01282 and PE/Dazzle 594; 60 ng/le6 cells BioLegend, 310941).

Protein production. HLA- ${ }^{\star} 01: 03$ HC residues $1-274$ and $\beta 2$-microglobulin $(\beta 2 \mathrm{M})$ were cloned into PET22b prokaryotic vectors and expressed in BL21 competent E.coli. Inclusion bodies were purified via sonication and homogenisation in a Triton-based buffer prior to final re-solubilisation in $8 \mathrm{M}$ urea, $50 \mathrm{mM}$ MES pH 6.5, 0.1 mM EDTA and 0.1 mM DTT ${ }^{23}$.

Peptide binding affinity assays. A peptide binding affinity assay was adapted from published micro-scale refold-ELISA-based methods ${ }^{8,39}$. In brief, $1 \mu \mathrm{M}$ HC and $1.5 \mu \mathrm{M}$ pre-refolded $\beta 2 \mathrm{M}$ were refolded in $0.33 \mathrm{mM}$ Tris-Maleate and $0.5 \%$ Lutrol-F68 in the presence of $70 \mu \mathrm{M}$ peptide, pre-diluted to $2 \mathrm{mM}$ working stocks in $100 \mathrm{mM}$ Tris-HCL pH 8. Micro-refolds were incubated at room temperature for $48 \mathrm{~h}$ before the relative capacity of each peptide to support stable HLA-E- $\beta 2 \mathrm{M}$ peptide complex formation was quantified by sandwich ELISA. Correctly refolded heterotrimeric complexes diluted 1:100 in 2\% IgG-free bovine serum albumin (BSA) were captured by the anti-human HLA-E monoclonal, 3D12 $(10 \mu \mathrm{g} / \mathrm{mL})$, in ELISA wells previously blocked and washed, respectively, with $2 \%$ BSA and $0.05 \%$ Tween-based wash buffer. $0.2 \mu \mathrm{g} / \mathrm{mL}$ polyclonal detection IgG raised in rabbits, specific for human $\beta 2 \mathrm{M}$ and enhancement antibodies specific for rabbit IgG, diluted 1:15 in $2 \%$ BSA, both conjugated to horseradish peroxidase, were sequentially added to ELISA wells to ensure detection of $\beta 2 \mathrm{M}$-associated forms of HLA-E only. Tetramethyl benzidine substrate and STOP solution were used to develop and terminate reactions, respectively, before obtaining absorbance readings at $450 \mathrm{~nm}$ on a FLUOstar OMEGA plate reader.

Protein refolding and purification. $\beta 2 \mathrm{M}$ (at a final concentration of $2 \mu \mathrm{M}$ ) was refolded in $100 \mathrm{mM}$ Tris pH8.0, 400mM L-arginine monohydrochloride, $2 \mathrm{mM}$ ethylenediamineteraacetic acid, $5 \mathrm{mM}$ reduced glutathione and $0.5 \mathrm{mM}$ oxidised Glutathione at $4{ }^{\circ} \mathrm{C}$ for $30 \mathrm{~min}$ before the addition of $20-50 \mu \mathrm{M}$ peptide. HLA$\mathrm{E}^{\star} 01: 03 \mathrm{HC}$ was pulsed into the refolding buffer until a final concentration of $1 \mu \mathrm{M}$ was reached. Following incubation for $72 \mathrm{~h}$ at $4{ }^{\circ} \mathrm{C}$, HLA-E refolds were filtered through $1.0 \mu \mathrm{m}$ cellular nitrate membranes to remove aggregates prior to concentration by centrifugation at $1000 \times g$ at $4{ }^{\circ} \mathrm{C}$ in Amicon Centricon Plus-70 and Ultra-15 10-kDa cut-off centrifugal filter devices. Samples were separated according to size into $20 \mathrm{mM}$ Tris $\mathrm{pH} 8,100 \mathrm{mM} \mathrm{NaCl}$ by fast protein liquid chromatography on a Superdex S75 16/60 column. Elution profiles were visualised by UV absorbance at $280 \mathrm{mAU}$, enabling differentiation of correctly refolded HLAE- $\beta 2 \mathrm{M}$-pepide complexes from smaller unassociated $\beta 2 \mathrm{M}$ and larger misfolded aggregates. Proteins were concentrated to $10 \mathrm{mg} / \mathrm{mL}$ for crystallisation and aliquots further analysed by SDS-PAGE electrophoresis to confirm presence of nonaggregated HLA-E HC and $\beta 2 \mathrm{~m}$.

Crystallisation screening. A total of $100 \mathrm{~nL}$ protein, at $10 \mathrm{mg} / \mathrm{mL}$, and $100 \mathrm{~nL}$ reservoir buffer were mixed in crystallisation wells and equilibrated by sitting drop vapour-diffusion at $20^{\circ} \mathrm{C}^{57}$. Commercial sparse matrix grid screens were used to identify optimal crystallisation conditions, around which ammonium sulphate fine gradient and additive screens were subsequently setup (crystallisation buffer conditions for the five structures reported here are specified in Supplementary Table 1). Crystals were cryopreserved in $25 \%$ glycerol and diffraction data were collected at Diamond Light Source Beamlines I04 and I24. Data collection statistics are listed in Supplementary Table 1.

Crystallographic analysis. Diffraction images from multiple isomorphous crystals were merged in Xia2 to increase completeness of the dataset ${ }^{58,59}$. Diffraction data were auto-indexed by Xia2 DIALS using the default parameters since 2015: I/sig(I) $>0.25$, merged $\mathrm{I} / \mathrm{sig}(\mathrm{I})>1$ and $\mathrm{CC} 1 / 2>0.5^{58-60}$. Initial phasing was carried out using the coordinates of the VL9-bound HLA-E*01:01 structure (PDB code 1MHE), stripped of peptide, hydrogens and waters as the search model in MolRep of the CCP4i suite ${ }^{61-63}$. Molecular replacement for RL9HIV and Mtb44 variant datasets was subsequently carried out in Phenix ${ }^{64}$ using the refined Mtb44 structure coordinates as the phasing model. Rigid body, restrained and TLS refinement were computed by CCP4i's REFMAC $5^{61}$ or Phenix.refine ${ }^{65}$ applying non-crystallographic symmetry restraints between iterative cycles of manual model building in $\operatorname{Coot}^{66}$. Models were validated using MolProbity ${ }^{67}$, visualised using the PyMOL Molecular Graphics System, version 2.0 (Schrödinger, LLC) and further investigated by PDBePISA ${ }^{68}$ and PDBeFOLD ${ }^{69}$.
Design of peptide- $\mathbf{\beta 2 m}$-HLA-E constructs. The coding sequence of mature HLA$\mathrm{E}^{\star} 01: 03 \mathrm{HC}$, previously mutagenised to incorporate a position Y84A mutation, was PCR amplified using the forward primer (that also included a synonymous change creating a BamH I restriction site), 5'-GACCTGGGCGGGaTCCCACTCCTTG AAGTATTTCC-3', and the reverse primer 5-gtggatcCAAGCTGTGAGACTCA GACCC- $3^{\prime 13,70}$. This construct was inserted into pEGFP-N1 downstream of a HindIII-BamH I cassette that contains the signal sequence of HLA-E*01:03, the coding sequence of the mature form of $\beta 2$-microglobulin and a flexible [GGGGS] linker. Wild type and position 2 (p2) mutants of the VL9 and Mtb44 peptide sequences, followed by a flexible [GGGGS $]_{3}$ linker, were introduced in between the HLA-E signal sequence and the start of the $\beta 2$-microglobulin sequence by overlap extension PCR

Peptide-\$2m-HLA-E transient transfection of 293 T cells. HEK 293T cells were maintained in $5 \% \mathrm{CO}_{2}$ in DMEM (Life Technologies) supplemented with $10 \%$ Foetal Bovine Serum (SeraLabs), and Penicillin/Streptomycin $(50$ and $50 \mu \mathrm{g} / \mathrm{mL}$, respectively, Life Technologies). Transfections were carried out at $70 \%$ confluency in six well plates using GeneJuice (Millipore) according to the manufacturer's instructions. Following $24 \mathrm{~h}$, cells were harvested. 1 million 293T cells were stained with $1 \mu \mathrm{L}$ of the anti-HLA-E monoclonal antibody, 3D12 (BioLegend) in $100 \mu \mathrm{l}$ PBS at $4{ }^{\circ} \mathrm{C}$ for $15 \mathrm{~min}$. Cells were washed twice with PBS and stained with secondary antibody (allophycocyanin-crosslinked Goat-Anti-Mouse $(\mathrm{H}+\mathrm{L}) \mathrm{F}\left(\mathrm{ab} \mathrm{b}^{\prime}\right) 2$ fragment [Life Technologies]) diluted 1:1500 in PBS for a further $15 \mathrm{~min}$. Subsequent to further (two) washing steps, cells were fixed in $100 \mu \mathrm{L}$ of Cytofix (BD Biosciences), and acquired using a CyAn ADP Analyser (Beckman Coulter). Transfected cells were gated according to light scatter (Forward versus Side) and EGFP + HLA-E/Mamu-E co-expression (Supplementary Fig. 1). Data analysis were performed using FlowJo (TreeStar) software. Four biological repeats were included per construct.

Thermal shift assay. The thermostability of refolded HLA-E- $\beta 2 \mathrm{~m}$ complexes was determined by heat-induced fluorescent dye incorporation, using the commercially available Protein Thermal Shift Dye Kit" (Applied Biosystems). In brief, $5 \mu \mathrm{g}$ of test HLA-E- $\beta 2 \mathrm{~m}$ complexes was aliquoted into $0.1 \mathrm{~mL}$ MicroAmp Fast Optical 96-well plates containing pre-mixed Protein Thermal Shift Dye and Protein Thermal Shift Buffer. Sample buffer (either PBS or Tris pH8, $100 \mathrm{mM} \mathrm{NaCL}$ ) was added to achieve a final volume of $20 \mu \mathrm{L}$. Control samples reconstituted with buffer were prepared to monitor background fluorescent signal. Both samples and controls were set up in quadruplicate. Thermal-driven dye incorporation was measured on an Applied Biosystem Real-Time 7500 Fast PCR System. Data was collected over a temperature ramp ranging from 25 to $95^{\circ} \mathrm{C}$, with $1{ }^{\circ} \mathrm{C}$ intervals. Melt curve data were analysed using Protein thermal Shift Software v1.3, and median Derivative Tm values $\left({ }^{\circ} \mathrm{C}\right)$ are reported.

Blue-native polyacrylamide gel electrophoresis(BN-PAGE). The composition of in vitro refolded HLA-E- $\beta 2 \mathrm{~m}$ complexes was evaluated using the Blue NativePAGE" Novex Bis-Tris gel system (life technologies), in accordance with the manufacturer's instructions (https://tools.thermofisher.com/content/sfs/manuals/ nativepage_man.pdf). In brief, $3 \mu \mathrm{L}$ of $4 \times$ Native-PAGE ${ }^{\prime \prime}$ Sample Buffer was added to $10 \mu \mathrm{g}(10 \mu \mathrm{L})$ of refolded HLA-E complexes, and immediately loaded on $3-12 \%$ Native-PAGE ${ }^{-\infty}$ Novex Bis-Tris gels. NativeMark ${ }^{\prime \prime \prime}$ Unstained Protein Standard was used as the ladder control. Gel electrophoresis was performed at 150 Volts (with current gradient from $15-16$ to $2-4 \mathrm{mAmps}$ ) for $2 \mathrm{~h}$ at room temperature. Following electrophoresis, gels were rinsed up to three times in MilliQ water prior to a $2-3 \mathrm{~h}$ staining step at room temperature in SimplyBlue ${ }^{\mathrm{mex}}$ SafeStain. De-staining was performed by multiple rounds of MilliQ water changes over a period of 24-48 h. Gel imaging was performed using a BioDoc IT Imaging System.

2D BN/SDS-PAGE analysis. 2D SDS-PAGE separation was applied to samples previously resolved by Native-PAGE ${ }^{\mathrm{w}}$ Novex Bis-Tris gel analysis. For the second dimension, SDS-PAGE NuPAGE 10\% Bis-Tris gels of standard 12-well lane gels were used for individual protein band evaluation. To analyse the composition of distinct native gel species, individual bands were carefully excised using Smart Slicer plastic razor blades (LevGo Inc.), then reduced (in 1× NuPage LDS sample buffer, $50 \mathrm{mM}$ DTT), alkylated (in $1 \times$ NuPage LDS with $50 \mathrm{mM}$ DMA) and quenched (in $1 \times$ NuPage LDS supplemented with $20 \%$ ethanol and $5 \mathrm{mM} \mathrm{DTT}$ ), prior to insertion into individual $1.5 \mathrm{~mm}$ wells of a 12-well NuPAGE gel. Electrophoresis was performed in MES buffer at a constant voltage of 200 for $35 \mathrm{~min}$ Following electroporation, NuPAGE gels were rinsed three times in milliQ water prior to a $2 \mathrm{~h}$ staining step in SimplyBlue ${ }^{\mathrm{m}}$ SafeStain. De-staining was performed in MilliQ water over a period of $24 \mathrm{~h}$. Gel imaging was performed using a BioDoc IT Imaging System.

Generation of HLA-E UV-labile monomers. Refolding of the VL9-based UVlabile 7MT2 peptide with HLA-E and $\beta 2 \mathrm{M}$, and subsequent purification was performed as outlined previously ${ }^{29}$. For UV photo-cleavage and peptide exchange, $0.5 \mu \mathrm{M}(\sim 25 \mathrm{mg} / \mathrm{mL})$ of UV-sensitive HLA-E-7MT2 monomer was incubated with $100 \mu \mathrm{M}$ "exchange" peptide in polypropylene V-shaped 96-well plates (Greiner Bio-One), and the final volume was adjusted to $125 \mu \mathrm{L}$ by adding exchange buffer 
(20 mM Tris, pH 7.4, $150 \mathrm{mM} \mathrm{NaCl}$ ). UV exchange samples were incubated for 60 min on ice in a Camag UV cabinet with a long-wave $366 \mathrm{~nm}$ UV lamp. Subsequent to photo-illumination, samples were centrifuged at $4000 \times g$ to remove aggregated material. To obtain sufficient material for BN-PAGE gel analysis, UV exchange reactions were set up in quadruplicate, and following the removal of protein aggregates as described above, samples were pooled and concentrated by centrifugation at $13,000 \times g$ in Vivaspin $5003 \mathrm{kDa}$ MWCO micro-concentrators (Sartorius) to a volume of $20 \mu \mathrm{L}$ prior to gel loading.

\section{Peptide exchange into pre-refolded HLA-E- $\beta 2 M-V L 9$ complexes and no} peptide refolded complexes. A total of $20 \mu \mathrm{g}$ of purified HLA-E- $\beta 2 \mathrm{~m}$ complexes, previously refolded without peptide or in the presence of VL9, were pulsed, respectively, with 50 or $200 \mathrm{M}$ excess of Mtb44 or RL9HIV peptides for $2 \mathrm{~h}$ at room temperature, in a final volume of $20 \mu \mathrm{L}$. Then, $10 \mu \mathrm{L}$ of each sample was loaded onto a BN-PAGE gel and their signature profiles were subsequently analysed.

Data availability. Structural factors and atomic coordinates have been deposited within the Protein Data Bank with acquisition codes: 6GH1, 6GH4, 6GGM, 6GHN and 6GL1.

All relevant data outlined in this study are available from the authors.

Received: 23 January 2018 Accepted: 4 July 2018

Published online: 07 August 2018

\section{References}

1. Boyson, J. E. et al. The MHC-E locus in macaques is polymorphic and is conserved between macaques and humans. Immunogenetics 41, 59-68 (1995).

2. Knapp, L. A., Cadavid, L. F. \& Watkins, D. I. The MHC-E locus is the most well conserved of all known primate class I histocompatibility genes. J. Immunol. 160, 189-196 (1998).

3. Pietra, G., Romagnani, C., Manzini, C., Moretta, L. \& Mingari, M. C. The emerging role of HLA-E-restricted CD8 $+\mathrm{T}$ lymphocytes in the adaptive immune response to pathogens and tumors. J. Biomed. Biotechnol. 2010, 907092 (2010).

4. Sullivan, L. C., Clements, C. S., Rossjohn, J. \& Brooks, A. G. The major histocompatibility complex class Ib molecule HLA-E at the interface between innate and adaptive immunity. Tissue Antigens 72, 415-424 (2008).

5. Braud, V., Jones, E. Y. \& McMichael, A. The human major histocompatibility complex class Ib molecule HLA-E binds signal sequence-derived peptides with primary anchor residues at positions 2 and 9. Eur. J. Immunol. 27, 1164-1169 (1997).

6. Lee, N. et al. HLA-E is a major ligand for the natural killer inhibitory receptor CD94/NKG2A. Proc. Natl Acad. Sci. USA 95, 5199-5204 (1998).

7. Lee, N., Goodlett, D. R., Ishitani, A., Marquardt, H. \& Geraghty, D. E. HLA-E surface expression depends on binding of TAP-dependent peptides derived from certain HLA class I signal sequences. J. Immunol. 160, 4951-4960 (1998).

8. Strong, R. K. et al. HLA-E allelic variants. Correlating differential expression, peptide affinities, crystal structures, and thermal stabilities. J. Biol. Chem. 278, 5082-5090 (2003).

9. Braud, V. M. et al. HLA-E binds to natural killer cell receptors CD94/NKG2A, B and C. Nature 391, 795-799 (1998).

10. Hoare, H. L. et al. Subtle changes in peptide conformation profoundly affect recognition of the non-classical MHC class I molecule HLA-E by the CD94NKG2 natural killer cell receptors. J. Mol. Biol. 377, 1297-1303 (2008).

11. Caccamo, N. et al. Human CD8 T lymphocytes recognize Mycobacterium tuberculosis antigens presented by HLA-E during active tuberculosis and express type 2 cytokines. Eur. J. Immunol. 45, 1069-1081 (2015).

12. Garcia, P. et al. Human T cell receptor-mediated recognition of HLA-E. Eur. J. Immunol. 32, 936-944 (2002).

13. Hansen, S. G. et al. Broadly targeted CD8(+) T cell responses restricted by major histocompatibility complex E. Science 351, 714-720 (2016)

14. Heinzel, A. S. et al. HLA-E-dependent presentation of Mtb-derived antigen to human CD8+ T cells. J. Exp. Med. 196, 1473-1481 (2002).

15. Joosten, S. A. et al. Mycobacterium tuberculosis peptides presented by HLA-E molecules are targets for human CD8 T cells with cytotoxic as well as regulatory activity. PLoS Pathog. 6, e1000782 (2010).

16. Lewinsohn, D. A. et al. Mycobacterium tuberculosis-specific CD8+ T cells preferentially recognize heavily infected cells. Am. J. Respir. Crit. Care Med. 168, 1346-1352 (2003).

17. Pietra, G. et al. HLA-E-restricted recognition of cytomegalovirus-derived peptides by human CD8+ cytolytic T lymphocytes. Proc. Natl Acad. Sci. USA 100, 10896-10901 (2003).
18. Pietra, G., Romagnani, C., Moretta, L. \& Mingari, M. C. HLA-E and HLA-Ebound peptides: recognition by subsets of NK and T cells. Curr. Pharm. Des. 15, 3336-3344 (2009)

19. Salerno-Goncalves, R., Fernandez-Vina, M., Lewinsohn, D. M. \& Sztein, M. B. Identification of a human HLA-E-restricted CD8+ T cell subset in volunteers immunized with Salmonella enterica serovar Typhi strain Ty21a typhoid vaccine. J. Immunol. 173, 5852-5862 (2004).

20. McMurtrey, C. et al. T cell recognition of Mycobacterium tuberculosis peptides presented by HLA-E derived from infected human cells. PLoS ONE 12, e0188288 (2017).

21. Harriff, M. J. et al. HLA-E presents glycopeptides from the Mycobacterium tuberculosis protein MPT32 to human CD8 ${ }^{+}$T cells. Sci. Rep. 7, 4622 (2017).

22. Grotzke, J. E. et al. The Mycobacterium tuberculosis phagosome is a HLA-I processing competent organelle. PLoS Pathog. 5, e1000374 (2009).

23. O'Callaghan, C. A. et al. Structural features impose tight peptide binding specificity in the non-classical MHC molecule HLA-E. Mol. Cell 1, 531-541 (1998).

24. Tomasec, P. et al. Surface expression of HLA-E, an inhibitor of natural killer cells, enhanced by human cytomegalovirus gpUL40. Science 287, 1031 (2000).

25. Ulbrecht, M. et al. Cutting edge: the human cytomegalovirus UL40 gene product contains a ligand for HLA-E and prevents NK cell-mediated lysis. J. Immunol. 164, 5019-5022 (2000).

26. Ulbrecht, M., Modrow, S., Srivastava, R., Peterson, P. A. \& Weiss, E. H. Interaction of HLA-E with peptides and the peptide transporter in vitro: implications for its function in antigen presentation. J. Immunol. 160, 4375-4385 (1998).

27. Lundegaard, C. et al. NetMHC-3.0: accurate web accessible predictions of human, mouse and monkey MHC class I affinities for peptides of length 8-11. Nucleic Acids Res. 36(Web Server issue), W509-W512 (2008).

28. Nattermann, J. et al. HIV-1 infection leads to increased HLA-E expression resulting in impaired function of natural killer cells. Antivir. Ther. 10, 95-107 (2005).

29. Toebes, M., Rodenko, B., Ovaa, H. \& Schumacher, T. N. Generation of peptide MHC class I monomers and multimers through ligand exchange. Curr. Protoc. Immunol. Chapter 18, Unit 1816 (2009).

30. Hansen, S. G. et al. Profound early control of highly pathogenic SIV by an effector memory T cell vaccine. Nature 473, 523-527 (2011).

31. Hansen, S. G. et al. Immune clearance of highly pathogenic SIV infection. Nature 502, 100-104 (2013).

32. Fruh, K. \& Picker, L. CD8+ T cell programming by cytomegalovirus vectors: applications in prophylactic and therapeutic vaccination. Curr. Opin. Immunol. 47, 52-56 (2017).

33. Romagnani, C. et al. HLA-E-restricted recognition of human cytomegalovirus by a subset of cytolytic T lymphocytes. Hum. Immunol. 65, 437-445 (2004).

34. Sztein, M. B. Cell-mediated immunity and antibody responses elicited by attenuated Salmonella enterica Serovar Typhi strains used as live oral vaccines in humans. Clin. Infect. Dis. 45, S15-S19 (2007).

35. Salerno-Goncalves, R., Wahid, R. \& Sztein, M. B. Ex vivo kinetics of early and long-term multifunctional human leukocyte antigen E-specific CD8+ cells in volunteers immunized with the Ty21a typhoid vaccine. Clin. Vaccine Immunol. 17, 1305-1314 (2010).

36. Goodridge, J. P., Burian, A., Lee, N. \& Geraghty, D. E. HLA-F complex without peptide binds to MHC class I protein in the open conformer form. J. Immunol. 184, 6199-6208 (2010).

37. Lampen, M. H. et al. Alternative peptide repertoire of HLA-E reveals a binding motif that is strikingly similar to HLA-A2. Mol. Immunol. 53, 126-131 (2013).

38. Wu, H. L. et al. The role of MHC-E in T cell immunity is conserved among humans, rhesus macaques, and cynomolgus macaques. J. Immunol. 200, 49-60 (2018)

39. Sylvester-Hvid, C. et al. Establishment of a quantitative ELISA capable of determining peptide-MHC class I interaction. Tissue Antigens 59, 251-258 (2002).

40. Borbulevych, O. Y., Baxter, T. K., Yu, Z., Restifo, N. P. \& Baker, B. M Increased immunogenicity of an anchor-modified tumor-associated antigen is due to the enhanced stability of the peptide/MHC complex: implications for vaccine design. J. Immunol. 174, 4812-4820 (2005).

41. Saini, S. K. et al. Dipeptides promote folding and peptide binding of MHC class I molecules. Proc. Natl Acad. Sci. USA 110, 15383-15388 (2013).

42. Saini, S. K. et al. Not all empty MHC class I molecules are molten globules: tryptophan fluorescence reveals a two-step mechanism of thermal denaturation. Mol. Immunol. 54, 386-396 (2013).

43. Fahnestock, M. L., Tamir, I., Narhi, L. \& Bjorkman, P. J. Thermal stability comparison of purified empty and peptide-filled forms of a class I MHC molecule. Science 258, 1658-1662 (1992).

44. Bouvier, M. \& Wiley, D. C. Importance of peptide amino and carboxyl termini to the stability of MHC class I molecules. Science 265, 398-402 (1994).

45. Kambayashi, T. et al. The non-classical MHC class I molecule Qa-1 forms unstable peptide complexes. J. Immunol. 172, 1661-1669 (2004). 
46. Lo Monaco, E. et al. HLA-E: strong association with beta2-microglobulin and surface expression in the absence of HLA class I signal sequence-derived peptides. J. Immunol. 181, 5442-5450 (2008).

47. Springer, S., Doring, K., Skipper, J. C., Townsend, A. R. \& Cerundolo, V. Fast association rates suggest a conformational change in the MHC class I molecule $\mathrm{H}-2 \mathrm{Db}$ upon peptide binding. Biochemistry 37, 3001-3012 (1998).

48. van Hateren, A., Bailey, A. \& Elliott, T. Recent advances in Major Histocompatibility Complex (MHC) class I antigen presentation: Plastic MHC molecules and TAPBPR-mediated quality control. F1000Research 6, 158 (2017).

49. Yanaka, S. et al. Peptide-dependent conformational fluctuation determines the stability of the human leukocyte antigen class I complex. J. Biol. Chem. 289, 24680-24690 (2014).

50. Jiang, J. et al. Crystal structure of a TAPBPR-MHC-I complex reveals the mechanism of peptide editing in antigen presentation. Science 358, 1064-1068 (2017).

51. Thomas, C. \& Tampe, R. Structure of the TAPBPR-MHC-I complex defines the mechanism of peptide loading and editing. Science 358, 1060-1064 (2017).

52. Boyle, L. H. et al. Tapasin-related protein TAPBPR is an additional component of the MHC class I presentation pathway. Proc. Natl Acad. Sci. USA 110, 3465-3470 (2013).

53. Blees, A. et al. Structure of the human MHC-I peptide-loading complex. Nature 551, 525-528 (2017).

54. Purbhoo, M. A., Irvine, D. J., Huppa, J. B. \& Davis, M. M. T cell killing does not require the formation of a stable mature immunological synapse. Nat. Immunol. 5, 524-530 (2004).

55. Theiler, J. et al. Epigraph: a vaccine design tool applied to an HIV therapeutic vaccine and a pan-filovirus vaccine. Sci. Rep. 6, 33987 (2016).

56. Hansen, S. G. et al. Evasion of CD8+ T cells is critical for superinfection by cytomegalovirus. Science 328, 102-106 (2010).

57. Walter, T. S. et al. A procedure for setting up high-throughput nanolitre crystallization experiments. Crystallization workflow for initial screening, automated storage, imaging and optimization. Acta Crystallogr. D Biol. Crystallogr. 61, 651-657 (2005).

58. Kabsch, W. Xds. Acta Crystallogr. D Biol. Crystallogr. 66, 125-132 (2010).

59. Evans, P. R. \& Murshudov, G. N. How good are my data and what is the resolution? Acta Crystallogr. D Biol. Crystallogr. 69, 1204-1214 (2013).

60. Karplus, P. A. \& Diederichs, K. Linking crystallographic model and data quality. Science 336, 1030-1033 (2012).

61. Winn, M. D. et al. Overview of the CCP4 suite and current developments. Acta Crystallogr. D Biol. Crystallogr. 67, 235-242 (2011).

62. Vagin, A. \& Teplyakov, A. Molecular replacement with MOLREP. Acta Crystallogr. D Biol. Crystallogr. 66, 22-25 (2010).

63. Murshudov, G. N. et al. REFMAC5 for the refinement of macromolecular crystal structures. Acta Crystallogr. D Biol. Crystallogr. 67, 355-367 (2011).

64. Adams, P. D. et al. PHENIX: a comprehensive python-based system for macromolecular structure solution. Acta Crystallogr. Sect. D Biol. Crystallogr. 66, 213-221 (2010).

65. Afonine, P. V. et al. Towards automated crystallographic structure refinement with phenix.refine. Acta Crystallogr. D Biol. Crystallogr. 68, 352-367 (2012).

66. Emsley, P., Lohkamp, B., Scott, W. G. \& Cowtan, K. Features and development of Coot. Acta Crystallogr. D Biol. Crystallogr. 66, 486-501 (2010).

67. Chen, V. B. et al. MolProbity: all-atom structure validation for macromolecular crystallography. Acta Crystallogr. D Biol. Crystallogr. 66, 12-21 (2010).

68. Krissinel, E. \& Henrick, K. Inference of macromolecular assemblies from crystalline state. J. Mol. Biol. 372, 774-797 (2007).

69. Krissinel, E. \& Henrick, K. Secondary-structure matching (SSM), a new tool for fast protein structure alignment in three dimensions. Acta Crystallogr. D Biol. Crystallogr. 60, 2256-2268 (2004).
70. Hansen, T. H., Connolly, J. M., Gould, K. G. \& Fremont, D. H. Basic and translational applications of engineered MHC class I proteins. Trends Immunol. 31, 363-369 (2010).

\section{Acknowledgements}

The authors would like to thank the research staff who collected study samples, and Diamond Light Source (Didcot, UK) for access to beamlines I04 and I21 (MX14744) that contributed to the results presented here. We also wish to thank Pamela Bjorkman, Tim Elliott and Ton Schumacher for helpful discussions, and David W. Morrow and Abigail B. Ventura for technical assistance. This work was supported by grants from the BMGF OPP1133649, NIH/NIAID UM1 AI 00645, NIH/NAID U19 AI 128741, UM1 AI 126619, MRC MR/K012037, and MRC MR/M019837/1. J.B.S. and S.G.H. are supported by grant R21 AI127125. L.J.P. is supported by grants from NIH, NIAID (P01-AI094417, R37AI054292, U19 AI095985, U19 AI096109, and P51-OD011092), and from the BMGF OPP1107409 and OPP1108533. V.J is funded by the EMBO long-term fellowship. E.Y.J. is supported by Cancer Research UK and MRC Programme Grants (C375/A17721 and MR/M000141/1), and Wellcome Trust grant 203141/Z/16/Z supporting the Wellcome Centre for Human Genetics.

\section{Author contributions}

L.C.W. performed binding and crystallographic experiments, interpreted data, and helped prepare the manuscript. K.H., V.J., D.R., T.S.W. and E.Y.J. helped with crystallographic data analysis/data interpretation. T.S.W. helped with crystallography screen set-up. S.B. and J.R.B. performed single-chain trimer experiments and data interpretation. C.O.'C. and P.B. helped with data evaluation. M.T. designed the UV peptide exchange peptide. S.G.H., S.A., J.M.G., J.S. and L.J.P. performed BCG vaccination studies, cellular experiments and helped with data interpretation. E.M. and K.F. constructed the RhCMV68-1/HIV vectors. A.J.Mc.M. and G.M.G. performed experiments, helped prepared the manuscript and supervised the project.

\section{Additional information}

Supplementary Information accompanies this paper at https://doi.org/10.1038/s41467 018-05459-z.

Competing interests: L.J.P., S.G.H. and K.F. have a significant financial interest in Vir Biotechnology, Inc., a company that may have a commercial interest in the results of this research and technology. The potential individual and institutional conflicts of interest have been reviewed and managed by OHSU. All other authors declare no competing interests.

Reprints and permission information is available online at http://npg.nature.com/ reprintsandpermissions/

Publisher's note: Springer Nature remains neutral with regard to jurisdictional claims in published maps and institutional affiliations.

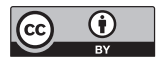

Open Access This article is licensed under a Creative Commons Attribution 4.0 International License, which permits use, sharing, adaptation, distribution and reproduction in any medium or format, as long as you give appropriate credit to the original author(s) and the source, provide a link to the Creative Commons license, and indicate if changes were made. The images or other third party material in this article are included in the article's Creative Commons license, unless indicated otherwise in a credit line to the material. If material is not included in the article's Creative Commons license and your intended use is not permitted by statutory regulation or exceeds the permitted use, you will need to obtain permission directly from the copyright holder. To view a copy of this license, visit http://creativecommons.org/ licenses/by/4.0/.

(C) The Author(s) 2018 